\title{
A RIEMANNIAN GEOMETRIC INVARIANT AND ITS APPLICATIONS TO A PROBLEM OF BOREL AND SERRE
}

\author{
BANG-YEN CHEN AND TADASHI NAGANO
}

\begin{abstract}
A new geometric invariant will be introduced, studied and determined on compact symmetric spaces.
\end{abstract}

Introduction. We will introduce a new invariant on Riemannian manifolds, which is especially interesting on compact symmetric spaces, and we will determine the invariant for the compact symmetric spaces, thus amplifying the announcement [CN1].

A symmetric space $M$ is defined with the point symmetry $s_{x}$ at every point $x$ of $M$. Our new invariant, denoted by $\#_{2} M$, may be defined as the maximal possible cardinality \# $A_{2}$ of a subset $A_{2}$ of $M$ such that the point symmetry $s_{x}$ fixes every point of $A_{2}$ for every $x$ in $A_{2}$. "The 2-number" $\#_{2} M$ is finite. $\#_{2} M$ is clearly equal to 1 if $M$ is not compact (but connected and simple). We thus consider compact spaces $M$ only. When $M$ is connected, the definition is equivalent to say that $\#_{2} M$ is the maximal possible cardinality \# $A_{2}$ of a subset $A_{2}$ of $M$ such that for every pair of points, $x$ and $y$, of $A_{2}$ there exists a closed geodesic of $M$ on which $x$ and $y$ are antipodal to each other. Thus the invariant could be defined on any connected Riemannian manifold.

It is easy to see (1.4) that the geometric invariant $\#_{2} M$ is a new obstruction to the existence of a totally geodesic embeddings $f: N \rightarrow M$, since the existence of $f$ clearly implies the inequality $\#_{2} N \leq \#_{2} M$. For example, while the complex Grassmann manifold $G_{2}\left(C^{4}\right)$ of the 2-dimensional subspaces of the complex vector space $C^{4}$ is obviously embedded into $G_{3}\left(C^{6}\right)$ as a totally submanifold, the space $G_{2}\left(C^{4}\right)^{*}$ which one obtains by identifying every member of $G_{2}\left(C^{4}\right)$ with its orthogonal complement in $C^{4}$, however, cannot be totally geodesically embedded into $G_{3}\left(C^{6}\right)^{*}$, because $\#_{2} G_{2}\left(C^{4}\right)^{*}=15>12=\#_{2} G_{3}\left(C^{6}\right)^{*}$ according to (6.4). The 2-number is not an obstruction to a topological embedding; for instance, the real projective space $G_{1}\left(R^{n}\right)$ can be topologically embedded in a sufficiently high dimensional sphere, but the 2-number $\#_{2} G_{1}\left(R^{n}\right)=n(>2)$ simply prohibits a totally geodesic embedding into any sphere whose 2 -number is 2 regardless of dimension.

Nevertheless, the invariant, $\#_{2} M$, has certain bearings on the topology of $M$ in other aspects; for instance, $\#_{2} M$ equals $\chi M$, the Euler number of $M$, if $M$ is a semisimple hermitian symmetric space (4.3); (in particular, one thus has $\chi M \geq \chi B$ for every hermitian subspace $B$ of a semisimple hermitian symmetric space $M$ ). And

Received by the editors March 11, 1985 and, in revised form, May 8, 1987.

1980 Mathematics Subject Classification (1985 Revision). Primary 53C21, 53C35, 22E40; Secondary $53 \mathrm{C} 40$.

Key words and phrases. Riemannian invariant, 2-rank, compact symmetric space, $\left(M_{+}, M_{-}\right)$method, Lie group, Euler characteristic, pole, centrosome. 
in general one has the inequality $\#_{2} M \geq \chi M$ for any compact connected symmetric space $M(4.1)$. To quote another example, let us mention a joint work of Borel and Serre [BS], from which our present work has originated. They considered the 2rank, $r_{2}$ (and $p$-rank for any prime number $p$ ) of a compact connected Lie group $G$, which is by definition the maximal possible rank of the elementary 2-subgroup, $\mathbf{Z}_{2} \times \cdots \times \mathbf{Z}_{2}$, of $G$. They proved: (i) the (usual) rank $r \leq r_{2} \leq 2 r$; and (ii) $G$ has (topological) 2-torsion if $r<r_{2}$. Now $G$ is a symmetric space, and it turns out (1.3) that $\#_{2} G$ is a power of 2 , in fact the $r_{2}$ th power of 2 . Our invariant generalizes their 2-rank in this sense, and their first estimate $r \leq r_{2}$ generalizes to $2^{r} \leq \#_{2} M$; see (1.9) for the second one. We do not have a generalization of (ii), but let us add that Takeuchi at Osaka proved $\#_{2}(M)=\operatorname{dim} H\left(M ; \mathbf{Z}_{2}\right)$ for any symmetric $R$-space $M$ in a personal communication; this formula is actually correct for every space we could check.

The full significance of our invariant is yet to be known, but we would like to mention briefly that the combinatorial geometry in the recent work of GelfandGoresky-MacPherson-Serganova (preprint) can be well understood with our invariant, or the maximal antipodal set $A_{2}$, together with Chow's arithmetic distance $[\mathbf{N}]$. There are several properties we believe are true and we cannot prove; for instance, the Euler number $\chi(M) \equiv \#_{2}(M)(\bmod 2)$ for every compact connected symmetric space $M$.

In this paper we assume a good knowledge of $[\mathbf{H}]$ on symmetric spaces on the part of the reader. In actual calculation of individual spaces, we need the fixed point sets of involutions on symmetric spaces. The reader may find it in the Appendix at the end of the paper.

Glossary of NOTATIONS. (I) Symmetric spaces. We use standard symbols (as in $[\mathbf{H}]$ ) to denote symmetric spaces, mostly. Here are a few minor exceptions. More specifically than $\mathrm{AI}, \mathrm{AI}(n)$ denotes $\mathrm{SU}(n) / \mathrm{SO}(n), \operatorname{AII}(n):=\mathrm{SU}(2 n) / \operatorname{Sp}(n)$, etc. $G_{d}\left(R^{n}\right), G_{d}\left(C^{n}\right)$ and $G_{d}\left(H^{n}\right)$ are the Grassmann manifolds of $d$-dimensional subspaces in the real, complex and quaternion vector spaces (or modules), respectively. Sometimes we write $G_{d}(n)$ for all these Grassmannians less specifically, when a statement with it is valid for all of them. $G_{d}^{o}\left(\mathbf{R}^{n}\right)$ denotes the Grassmann manifold of the oriented $d$-dimensional subspaces of $\mathbf{R}^{n} . M^{\sim}$ and $M^{*}$ denote the universal covering space and the bottom space (the adjoint space in $[\mathbf{H}]$. See $3.1 \mathrm{a}$ ) of the space $M . M / \mathrm{Z} \mu$ denotes the space of which $M$ is a covering space with the covering transformation group $\mathbf{Z} \mu$, the cyclic group of order $\mu$ (if there is no ambiguity). The standard notations for the exceptional spaces such as $G_{2}, F_{4}, E_{6}, \ldots$, GI, . , EIX denote the 1-connected spaces, where we write GI for $G_{2} / \operatorname{SO}(4)$. Groups $D[4]$ and $Q[8]$ are defined in 1.5 .

(II) Matrices. $\mathrm{I}_{m}$ is the diagonal and orthogonal matrix whose first $m$ diagonal entries are -1 and the other diagonal entries are 1. $\kappa$ [resp. $\iota]$ denotes the mapping which carries a matrix in $\mathrm{U}(n)$ into its complex conjugate [resp. inverse]. The symbol $\iota$ also denotes the mapping which carries a member of a group into its inverse. Let $\left(\varepsilon_{1}, \ldots, \varepsilon_{n}\right)$ be a fixed orthonormal basis for the metric cartesian space $\mathbf{C}^{n}$ (and $\mathbf{R}^{n}$ ). In case $n=2 n^{\prime}$ is even, $J$ or $J_{n^{\prime}}$ denotes "the complex structure", $J^{2}=-1$, such that $J\left(\varepsilon_{i}\right)=\varepsilon_{i+n^{\prime}}, 1 \leq i \leq n^{\prime}$, while $K$ or $K_{n^{\prime}}$ denotes the involution $\left(K^{2}=1\right)$ satisfying the same equalities $K\left(\varepsilon_{i}\right)=\varepsilon_{i+n^{\prime}}$ for $i=1, \ldots, m$. 
1. Symmetric spaces and $\#_{2} M$. We introduce basic concepts, followed by examples and comments. Let $M$ be a compact (Riemannian) symmetric space (see $[\mathbf{H}]$ ); thus there is a symmetry $s_{x}$ of $M$ at each point $x$ of $M$, such that $x$ is isolated in the fixed point set, $F\left(s_{x}, M\right)$, of $s_{x}$. There is a Riemannian metric $g$ for which all the symmetries are isometries. We fix $g$ for convenience, although $g$ is not unique. A smooth map $f: X \rightarrow Y$ of a symmetric space $X$ into another is called a morphism if $f$ commutes with the symmetries; $s_{f(x)} \circ f=f \circ s_{x}$ for $x$ in $X$ (cf. [N]).

A discrete manifold $\Sigma$ becomes a symmetric space by choosing the identity map as the symmetry at every point. We call $\Sigma$ a trivial symmetric space.

DEFINITIONS 1.1. \# $2 M$, the 2-number of $M$, is the supremum of the cardinalities, \# $\Sigma$, of the finite trivial spaces $\Sigma$ which admit monomorphisms into $M$. In other words, $\#_{2} M$ is the supremum of the cardinalities of the subsets $A_{2}$ of $M$ such that $s_{x}$ fixes every point of $A_{2}$ for every point $x$ of $A_{2}$. Such a subset $A_{2}$ is called an antipodal set in $M$. For brevity, we call an antipodal set in $M$ great if its cardinality equals $\#_{2} M$. The great ones are not unique up to congruence.

REMARK 1.2. \# ${ }_{2} M$ is finite, since every $x$ is isolated in $F\left(s_{x}, M\right)$.

REMARK 1.3 . If $M$ is a compact Lie group, then $\#_{2} M$ is closely related to the 2-rank of $M$, [BS and Q]. We will explain this. A compact Lie group $M$ becomes a symmetric space by assigning $s_{x}(y):=x y^{-1} x$ to every point $x$. The automorphism group $\operatorname{Aut}(M)$ of the space $M$ contains the left translation group, and hence $\operatorname{Aut}(M)$ is transitive on $M$. In finding $\#_{2} M$, we may therefore assume that a maximal antipodal set $A_{2}$ in $M$ contains the unit element 1 . First observe that the fixed point set $F\left(s_{1}, M\right)$ consists of the members of order 1 or 2 . Thus we have $s_{x}(y)=y$ for two members $x, y$ of $A_{2}$ if and only if $x y=y x$. Also observe that this implies that the maximal $A_{2}$ is a subgroup. Moreover $A_{2}$ is an elementary abelian 2-subgroup $\cong\left(\mathbf{Z}_{2}\right)^{t}$, a 2-subgroup for short. The largest possible value of $t$ is by definition the 2-rank of $M$, we denote it by $r_{2}(M)$. In particular $\#_{2} M$ is a power of 2 for a group $M$. It is known [B2] that the cohomology of $M$ has no 2 -torsion if and only if every antipodal subgroup $A_{2}$ is contained in some torus in $M$, provided $M$ is connected. Furthermore [B2], if the group $M$ is 1-connected and $M$ does have a 2 -torsion, then $M$ contains an antipodal group of rank 3 which no torus of $M$ contains.

REMARK 1.4. The 2-number gives an obstruction to the existence of a monomorphism $f: M^{\prime} \rightarrow M$. In fact if $f$ exists, it follows immediately from the definitions that $f$ carries every antipodal set in $M^{\prime}$ onto an antipodal set in $M$ and hence one has $\#_{2} M^{\prime} \leq \#_{2} M$ necessarily. The 2 -number also gives a necessary condition for totally geodesic embeddings (see 2.1).

EXAMPLES 1.5. We denote by $D[4]$ the dihedral group of order 8 , or the automorphism group of a square in the plane. Thus $D[4]$ is generated, say, by the reflections in the $x$-axis and the line $y=x$ in the Euclidean plane. Clearly $\#{ }_{2} D[4]=$ 4. Let $Q[8]$ denote the quaternion group, generated, by $i$ and $j$ in the group of the nonzero quaternions, where $i$ and $j$ together with $k$ form a standard basis for the pure quaternions. One has $\#{ }_{2} Q[8]=2$. Their commutator subgroups have the 2 -numbers both equal to 4 . These groups will be useful later (5.15 among others) because of the following characterizations. If a group contains two distinct members $a, b$ satisfying $a^{2}=1, b^{2}=1$ (resp. $a^{2}=-1, b^{2}=-1=$ an involutive member in the center) and the commutator $[a, b]=-1$, then $a$ and $b$ generate a subgroup 
which is isomorphic with $D[4]$ (resp. $Q[8]$ ). Later, we will use representations of these groups, in which -1 will act on the vector spaces as -1 .

EXAMPLES 1.6. The sphere has $\#_{2} S^{n}=2$ obviously. The $r$-dimensional torus $T^{r}$ has $\#_{2} T^{r}=2^{r}$ by the next lemma.

LEMMA 1.7. We have $\#_{2}(M \times N)=\left(\#_{2} M\right)\left(\#_{2} N\right)$ for the product of compact symmetric spaces $M$ and $N$.

PrOOF. The symmetry at $(x, y)$ in $M \times N$ carries a point $(u, v)$ into $\left(s_{x} u, s_{y} v\right)$. Hence $F\left(s_{(x, y)}, M \times N\right)=F\left(s_{x}, M\right) \times F\left(s_{y}, N\right)$.

If $A_{2}$ is a great antipodal set in $M$, then $A_{2}$ is contained in the subspace $F\left(s_{o}, M\right)$ for any point $o$ in $A_{2}$ and therefore $\#_{2} M=\#_{2} F\left(s_{o}, M\right)$ by 1.4. Compare this with Proposition 1.9 below, however.

DEFINITION 1.8. A polar of $o$ in $M$ is a connected component $\neq\{o\}$ of $F\left(s_{o}, M\right)$ and is denoted by $M^{+}, M^{+}(p)$ or $M^{+}(p ; o)$ if it contains $p$ in it. Every polar is a subspace.

PROPOSITION 1.9. \# ${ }_{2} M-1$ does not exceed the sum of the 2-numbers of all the polars of a point in $M ; \#_{2} M-1 \leq \Sigma \#_{2} M^{+}$.

ProOF. Let $A_{2}$ be a great antipodal set in $M$. Let $o$ be a point of $A_{2}$. Then $A_{2}$ is a subspace of $F\left(s_{o}, M\right)$, of which $\{o\}$ is a connected component. The intersection of $A_{2}$ with each polar $M^{+}$is antipodal in it. And the inequality is proven.

REMARK 1.10. The equality in 1.9 holds in many cases (such as the groups $\mathrm{Sp}(n)$ and $\mathrm{O}(n)$ and the hermitian symmetric spaces) and does not in the other cases (such as the adjoint group $\mathrm{SU}(8)^{*}$ of $\mathrm{SU}(8)$ ). See (2.10) through (2.12) and (3.13) for a few more examples. It is an interesting problem to determine exactly when the equality in 1.9 is valid.

2. Connected spaces and the 2-number. From now on, we assume that the compact symmetric space $M$ is connected. We will relate basic concepts introduced in the previous section to Riemannian geometry. In particular, we will use the next lemma to characterize the antipodal sets geometrically.

LEMMA 2.1. A smooth map $f: M \rightarrow M^{\prime \prime}$ is a morphism if and only if $f$ is totally geodesic.

ProOF. Assume that $f$ is totally geodesic, that is, $f$ carries the geodesics in $M$ into geodesics in $M^{\prime \prime}$ preserving the affine parameters. Then $f$ is a linear map with respect to normal coordinate systems centered at any point $x$ of $M$ and at $f(x)$ in $M^{\prime \prime}$. Since $s_{x}$ and $s_{f(x)}$ are -1 times the identity in these coordinates, we have $f \circ s_{x}=s_{f(x)} \circ f$ on the coordinate neighborhood. This equality is valid globally on $M$, since $M$ is connected and complete. Therefore $f$ is a morphism. Conversely, assume that $f$ is a morphism. First we consider the case where $\operatorname{dim} M$ is one and $M$ is not necessarily compact. Then $M$ is an abelian group with the symmetries in (1.3) and the identity map $1_{M}$ is an affine parameter. Since $f$ is a morphism, the image $f(M)$ is stabilized by the symmetry $s_{f(x)}$ at every point $f(x)$ of $f(M)$. In particular $s_{f(x)}$ fixes the first normal vector (the covariant derivative of the tangent vector to the curve $f), f^{N}$. Thus $f^{N}=0$, since $s_{f(x)}$ is -1 on the tangent space $T_{f(x)} M^{\prime \prime}$. Therefore $f(M)$ is a geodesic. Moreover $f$ preserves the affine parameter, since $f$ is a morphism. In the general case of $\operatorname{dim} M>1$, the above argument easily shows that $f$ is totally geodesic. 
COROLLARY 2.2. The automorphism group of $M$ contains the isometry group $I(M, g)$, which in turn contains the symmetries.

REMARK 2.3. The identity component of $I(M, g)$, denoted by $G, G_{M}$ or $G(M)$, is then transitive on $M$. Hence we may assume that an antipodal set $A_{2}$ in consideration contains a chosen point $o$ whenever convenient. $G$ is actually independent of $g$.

PROPOSITION 2.4. A subset $A_{2}$ of a connected symmetric space $M$ is antipodal if and only if any pair of points in $A_{2}$ are antipodal points on some circle $T=S^{1}$ (which is of course a subspace and hence a geodesic).

PROOF. The crucial point is to show that every morphism of the trivial space $\{a, b\}$ of two points into a connected space $M$ extends to a morphism from a circle $T$. Now take any pair of points, $\{o, p\}$, in $A_{2}$. Then there is a maximal torus $A$ in $M$ which contains the two points $o, p$. The pair $\{o, p\}$ is antipodal in $A$ obviously. Thus $o, p$ are antipodal points on a circle $T$ in $A$ (and hence in $M$ ). The converse is easy.

REMARK 2.5. Proposition 2.4 suggests that the 2-number can be defined and studied for any compact connected Riemannian manifold. But we will not pursue it in this paper.

REMARK 2.6. (2.4) also shows that the concept of the polar, $M^{+}(p)$, is the same as that of $M^{+}(p)$ in $[\mathbf{C N}]$. The polars have been completely determined for every connected irreducible symmetric space in [CN and $\mathbf{N}]$. We will list the results in the appendix, since we will need them in later sections. To illustrate a geometric meaning of polars, let us notice that a (complete) geodesic passing through $o$ is closed if and only if it meets a polar of $o$.

PROPOSITION 2.7. We have $2^{r(M)} \leq \#_{2} M$, where $r(M)$ is the rank of $M$.

ProOF. Immediate from (1.4) and (1.6).

DEFINITION 2.8. Cartan's quadratic morphism $Q=Q_{o}: M \rightarrow G$ carries a point $x$ into $s_{x} s_{o} . Q$ is a $G$-equivariant morphism which is an immersion, where, in the action of $G$ on itself, $b \in G$ caries $c \in G$ into $b c \sigma(b)^{-1}$. The points $\neq o$ mapped to the identity are called the poles of $o$ in $M$. The poles are polars.

PROPOSITION 2.9. The following 6 conditions are equivalent to each other for two distinct points $o, p$ of a connected space $M$. (i) $p$ is a pole of $o$ in $M$; (ii) $s_{p}=s_{o}$; (iii) $\{p\}$ is a polar of $o$ in $M$; (iv) there is a double covering morphism $\pi=\pi_{\{o, p\}}: M \rightarrow M^{\prime \prime}$ with $\pi(p)=\pi(o) ;(\mathrm{v}) p$ is a point in the orbit $F(\sigma, G)(o)$ of the group $F(\sigma, G)$ through $o, \sigma:=\operatorname{ad}\left(s_{o}\right)$, and (vi) the isotropy subgroup of $S G$ at $p$ is that, $S K$, of $S G$ at $o$, where $S G$ is the group generated by $G$ and the symmetries; $S G / G$ is a group of order $\leq 2$.

ProOF. That (i) $\Leftrightarrow$ (ii) is immediate from the definition. (ii) $\Rightarrow($ iii), since $p$ is isolated in $F\left(s_{p}, M\right)=F\left(s_{o}, M\right)$, and vice versa; (ii) $\Leftrightarrow$ (iii). Recall every covering morphism of a symmetric space is regular and its covering transformation group is abelian. In particular, $Q: M \rightarrow Q(M)$ is the projection of $M$ onto the orbit space of the covering transformation group $\Gamma \subset I(M)$. Now assume (i). Then some member $\gamma$ of $\Gamma$ carries $o$ into $p$. On the other hand, $G$ contains an involutive member $c$ which 
carries $o$ into $p$ as well. Since $\Gamma$ centralizes $G$, we see $\gamma p=\gamma c(o)=c \gamma(o)=c(p)=$ $c^{2}(o)=o$. Therefore the free transformation $\gamma$ is involutive. And we conclude (iv) by choosing $M^{\prime \prime}:=M /\{1, \gamma\}$, the orbit space; thus (i) $\Rightarrow$ (iv). In passing, we note that we may assume $\sigma(c)=c^{-1}(=c)$. And (iv) implies (v). Assume (v); that is, $p=c(o)$ for some $c$ in $G$ which is fixed by $\sigma$. Then $s_{p}=s_{c(o)}=c s_{o} c^{-1}=s_{o}$ by $\sigma(c)=c$. Thus (v) $\Rightarrow(\mathrm{ii})$, and hence (i) $\Leftrightarrow(\mathrm{ii}) \Leftrightarrow(\mathrm{iii}) \Leftrightarrow(\mathrm{iv}) \Leftrightarrow(\mathrm{v})$. We will conclude (vi) from these equivalent conditions. Since $Q$ is $G$-equivariant, the isotropy subgroup $K$ acts on $M^{\prime \prime}$. $K$ fixes $o^{\prime \prime}:=\pi(o)$ on $M^{\prime \prime}$. Hence $K$ stabilizes $\{o, p\}=\pi^{-1}\left(o^{\prime \prime}\right)$ on $M$. Since $K$ fixes $o, K$ therefore fixes $p$, and (vi) is proven. Finally assume (vi). Then $s_{o} \in S K$ fixes $p$ and the polar $S K(p)$ is a singleton $\{p\}$. (Recall from [CN] that the $S K$-orbit through a point fixed by $s_{o}$ is a polar.) Thus we have (iii). The proof of the proposition is now complete.

As a simple application of the concept of the pole, we will prove the next proposition concerning the equality in 1.9 .

Proposition 2.10. The equality $\#_{2} M-1=\Sigma \#_{2} M^{+}$holds in 1.9 if all but one of the polars of $o$ in $M$ are poles.

PROOF. The equality is valid if the symmetry $s_{x}$ at every point $x$ in every polar $M^{+}(p)$ of $o$ is the identity on every other polar, since the disjoint union of $\{o\}$ and great antipodal sets in the polars, chosen one from each polar, will be a great one in $M$. Lemma 2.9 says that this condition is satisfied.

EXAMPLE 2.11. A projective space of "dimension" $n$ has $\#_{2} M=n+1$; namely, $G_{1}\left(R^{n+1}\right), G_{1}\left(C^{n+1}\right)$ and $G_{1}\left(H^{n+1}\right)$ have $\#_{2}=n$, and $\#_{2}(\mathrm{FII})=1+\#_{2}\left(S^{8}\right)=3$.

ExAMPLE 2.12. \# ${ }_{2} \mathrm{EIV}=1+\#_{2} \mathrm{FII}=4$ by $(2.10)$ and $(2.11)$, since FII is the only polar in EIV.

REMARK 2.13. Let $o$ and $p$ be points in an antipodal set $A_{2}$ in $M$. Then $A_{2}$ is contained in the fixed point set $F(Q(p), M)$ obviously. Thus, if $A_{2}$ is great, then one has $\#_{2} M=\#_{2} F(Q(p), M)$. The point $p$ lies in a polar $M^{+}(p)$ of $o$ in $M$. The orthogonal space $M^{-}(p)$ to $M^{+}(p)$ at $p$ is by definition the connected subspace whose tangent space at $p$ is the orthogonal complement of the tangent space to $M^{+}(p)$ in $T_{p}(M) . M^{-}(p)$ is the connected component of $F(Q(p), M)$ through $p$. One has $M^{-}(p)=F(Q(p), M)$ if $M$ is a 1-connected group, as is well known. Thus, if $A_{2}$ is a great antipodal subgroup of a 1-connected group $M$ and if $A_{2}$ meets $M^{+}(p)$, then the 2-rank of $M$ equals that of the subgroup $M^{-}(p)$. In particular, it equals the 2-rank of at least one of the subgroups which are the orthogonal spaces to the polars in $M$. This easy fact will be used in later sections.

LEMMA 2.14. Every maximal torus $A$ of a connected symmetric space $M$ meets every polar of a point $O$ in $A$.

PROOF. A point of a polar of $o$ is its polar in a circle passing through it and $o$. A member of the isotropy subgroup $K$ at $o$ carries the circle into $A$ (as is well known $[\mathbf{H}])$. And $K$ stabilizes every polar $[\mathbf{C N}]$.

REMARK 2.15. A maximal or even great antipodal set $A_{2}$ does not necessarily meet every polar of a point in it, however.

3. Covering maps and $\#_{2} M$.

Proposition 3.1. One has $\#_{2} M^{\prime}=\#_{2} M$, if there exists a $k$-fold covering morphism $f: M^{\prime} \rightarrow M$ and $k$ is odd. 
PROOF. Since the fundamental groups of all the compact symmetric spaces are abelian, the structure group of the fibration $f$ is the covering transformation group $\Gamma$ and we assume that $\Gamma$ is a cyclic group of order $k$ and that $k$ is prime. Then the fibre $f^{-1}(x)$ of each point $x$ of $M$ is contained in a circle $c$. In particular no two points in $f^{-1}(x)$ are antipodal to each other, since $k$ is odd. This implies $\#_{2} M^{\prime} \leq \#_{2} M$; in fact an antipodal set $A_{2}^{\prime}$ in $M^{\prime}$ projects onto an antipodal set $A_{2}$ in $M$ under $f$ which certainly carries circles onto circles and moreover the restriction of $f$ to $A_{2}^{\prime}$ is injective as we have just seen. For the converse or the opposite inequality, we first consider two points $x, y$ in a given antipodal set $A_{2}$ in $M$. We wish to show that a lift $x^{\prime} \in f^{-1}(x)$ of $x$ is antipodal to exactly one lift $y^{\prime}$ of $y$. Let $c$ be a circle in $M^{\prime}$ which contains $f^{-1}(x)$ in it. Then $s_{x^{\prime}}$ stabilizes $c$, since $s_{x}$ fixes $y=f\left(y^{\prime}\right)$ and stabilizes the circle $f \circ c$ which passes through $y$. If $s_{x^{\prime}}$ reverses the orientation of $c$, then $s_{x^{\prime}}$ fixes exactly one point in $f^{-1}(y) \subset c$, since $k$ is odd. If $s_{x^{\prime}}$ preserves it on the contrary, then $s_{x^{\prime}}$ is the identity on $c$, which is impossible for the following reason. The circle $c$ is then contained in a polar $M^{\prime+}\left(y^{\prime}\right)$ of $x^{\prime}$ in $M^{\prime}$. On the other hand, the orthogonal space $M^{\prime-}\left(y^{\prime}\right)$ is a $k$-fold covering space of the projection $M^{-}(y)$ under $f$, since the orthogonal spaces to the polars contain the maximal tori in the whole space in general. Thus there is a circle $d$ in $M^{\prime-}\left(y^{\prime}\right)$ which contains $f^{-1}(y)$. Then $d$ is contained in $M^{\prime+}\left(y^{\prime}\right)$ according to the above, which is absurd, since $M^{\prime-}\left(y^{\prime}\right)$ is orthogonal to $M^{\prime+}\left(y^{\prime}\right)$ at $y^{\prime}$. Therefore $s_{x^{\prime}}$ fixes exactly one point in $f^{-1}(y)$ if $f\left(x^{\prime}\right)$ and $y^{\prime}$ sit in $A_{2}$. Finally let $x, y, z$ be points in $A_{2}$ and assume that a lift $x^{\prime}$ of $x$ is antipodal to $y^{\prime}$ and to $z^{\prime}, f\left(y^{\prime}\right)=y$ and $f\left(z^{\prime}\right)=z$. It remains to show that $y^{\prime}$ is antipodal to $z^{\prime}$. We have $s_{y^{\prime}} z^{\prime}=\gamma z^{\prime}$ for some covering transformation $\gamma \in \Gamma$. Thus $s_{x^{\prime}} \gamma z^{\prime}=s_{x^{\prime}} s_{y^{\prime}} z^{\prime}=s_{y^{\prime}} s_{x^{\prime}} z^{\prime}=s_{y^{\prime}} z^{\prime}=\gamma z^{\prime}$. Since $s_{x^{\prime}} z^{\prime}=z^{\prime}$, this gives $\gamma z^{\prime}=z^{\prime}$ by uniqueness, that is, $s_{y^{\prime}} z^{\prime}=\gamma z^{\prime}$. Therefore some antipodal set $A_{2}^{\prime}$ in $M^{\prime}$ projects onto the given antipodal set $A_{2}$ in $M$ under $f$.

DEFINITION 3.1a. Every local isomorphism class of connected semisimple symmetric spaces contains a unique space $M^{*}$ of which every other space in the class is a covering space. We call $M^{*}$ the bottom space in the class or that of any space in it. $\left(M^{*}\right.$ is called the adjoint space in $[\mathbf{H}]$.)

COROLlaRY 3.2. \# ${ }_{2} M$ depends only on the local class of $M$ if $M$ is one of the spaces, $\mathrm{SU}(k), \mathrm{AI}(k), \mathrm{AII}(k), E_{6}, \mathrm{EI}$ and $\mathrm{EIV}$, where $k$ is odd.

PROOF. The fundamental groups of the bottom spaces $M^{*}$ have odd orders.

Because of 3.1, we will concentrate on double covering morphisms $\pi: M \rightarrow M^{\prime \prime}$. There is a pole $p$ of $o$ in $M$ which goes to $\pi(o)=\pi(p)$ under $\pi$. In passing to $M^{\prime \prime}$, the polar $\{p\}$ is lost and new ones are created; the next definition of the centrosome (a term borrowed from biology) may be used to articulate this situation.

DEFinition 3.3. The centrosome $C(o, p)=C(o, p ; M)$ for the pair $(o, p)$ of a point $o$ and its pole $p$ in $M$ is the subset consisting of the midpoints $x$ of the geodesics from $o$ to $p$.

In other words, $C(o, p)$ is the complement $\pi^{-1}\left(F\left(s_{o^{\prime \prime}}, M^{\prime \prime}\right)\right) \backslash F\left(s_{o}, M\right)$, where $o^{\prime \prime}=\pi(o)$. Hence $C(o, p)$ is a subspace, which may be disconnected. We will establish its basic properties.

PROPOSITION 3.4. The following 5 conditions are equivalent to each other for any two distinct points $o, q$ in $M$ : (i) $s_{o} s_{q}=s_{q} s_{o}$; (ii) $Q(q)^{2}=1_{M}$; (iii) either $s_{o}$ fixes $q$ or $q$ is a point in the controsome $C(o, p)$ for some pole $p$ of $o$; (iv) either 
$s_{o}(q)=q$ or $s_{o}(q)=\gamma(q)$ for the covering transformation $\gamma$ for some pole $p=\gamma(o)$ of o (see 2.9); and $(\mathrm{v})$ either $s_{o}(q)=q$ or there is a double covering morphism $\pi$ : $M \rightarrow M^{\prime \prime}$ such that $s_{o^{\prime \prime}}$ fixes $q^{\prime \prime}$ where $o^{\prime \prime}=\pi(o)$ and $q^{\prime \prime}=\pi(q)$.

ProOF. That (i) $\Leftrightarrow$ (ii) is immediate from Definition 2.8. We may assume $s_{o}(q) \neq$ $q$, since otherwise all the 5 conditions are satisfied. We have $s_{q}(o) \neq o$ too. Assume (i). Then the symmetry at $p:=s_{q}(o)$ is $s_{o}$, since $s_{q}$ is an automorphism of $M$. Therefore $p$ is a pole of $o$ by 2.9 and $p \neq o$. For any geodesic arc $c$ from $q$ to $o$, $q$ is the midpoint of the geodesic arc $c \cup s_{q}(c)$ joining $o$ and $p$. Hence (i) $\Rightarrow$ (iii). Assume (iii). Extend $c$ to a circle $T$. Project $T$ into $M^{\prime \prime}$ under the double covering morphism $\pi$ which corresponds to the pair $(o, p)$ by 2.9. The image is a circle. In other words, the restriction of $\pi$ to $T$ is also a double covering morphism. Therefore $\gamma$ stabilizes $T$. Now it is obvious that $\gamma(q)$ is $s_{o}(q)$, showing (iii) $\Rightarrow$ (iv). (v) follows from (iv) easily. Finally assume (v). Then $o^{\prime \prime}$ and $q^{\prime \prime}$ are antipodal points on a circle $T^{\prime \prime}$ in $M^{\prime \prime}$. Its lift $T$ in $M$ passes through $o$ and $q$. The antipodal point $p$ of $o$ in $T$ is a pole of $o$ in $M$ by 2.9. Thus $Q(q)$ is the antipodal point of $Q(o)=1$ on the circle $Q(T)$, which is contained in the group $G$. Therefore $Q(q)$ is involutive (see 1.3), and we have (ii).

PROPOSITION 3.5. The equality is valid in 1.9, if there are exactly two polars of positive dimensions and if they project onto a single polar under a double covering morphism.

PROOF. Let $\gamma$ be the corresponding covering morphism. Let $A_{2}$ be an antipodal set in $M^{+}$, a polar of $o$ with $\operatorname{dim} M^{+}>0$. Consider the union $U$ of $\{o\}$, \{the poles of $o\}, A_{2}$ and $\gamma\left(A_{2}\right)$. We will show that $\mathrm{U}$ is antipodal in $M . \gamma\left(A_{2}\right)$ is antipodal in the polar $\gamma\left(M^{+}\right)$, since $\gamma$ is an automorphism of $M . A_{2} \cup \gamma\left(A_{2}\right)$ is antipodal in $M$, since $s_{x}(\gamma(y))=\gamma s_{x}(y)=\gamma y$ for points $x, y$ in $A_{2}$. Thus $\mathrm{U}$ is antipodal by the property (ii) in (2.9). Hence we have the opposite inequality $\#_{2} M-1 \geq \#$ the poles of $o\}+2 \#_{2} M^{+}$, which proves the proposition together with 1.9.

EXAMPLE 3.6. The group $E_{7}$ fits 3.5 with $M^{+}=E V I$ and a single pole. Thus $\#_{2} E_{7}=2\left(1+\#_{2} \mathrm{EVI}\right)$. Similarly $\#_{2} \mathrm{EV}=2\left(1+G_{4}\left(C^{8}\right)^{*}\right)$. Also $\#_{2} \mathrm{EVII}=$ $2\left(1+\#_{2}\right.$ EIII $)$.

PROPOSITION 3.7. If $M$ is a double covering space of $M^{\prime \prime}$, then we have $\#_{2} M \leq 2 \#_{2} M^{\prime \prime}$. (The equality is true in case $M$ is $\mathrm{SO}(2 m), 2 m>4$; see 5.12.)

ProOF. Let $A_{2}$ be a maximal antipodal set in $M$. The union $A_{2} \cup \gamma\left(A_{2}\right)$ is also antipodal, where $\gamma$ is the covering transformation for the covering morphism $\pi: M \rightarrow M^{\prime \prime}$. That is, $\gamma$ stabilizes $A_{2} . \pi\left(A_{2}\right)$ is antipodal in $M^{\prime \prime}$. Therefore $\#\left(A_{2}\right)=2 \#\left(\pi A_{2}\right) \leq 2 \#{ }_{2} M^{\prime \prime}$.

DEFINITION AND NOTATION 3.8. Suppose a finite group $\Gamma$ is acting on two spaces $M$ and $N$ freely as automorphism groups. Then $\Gamma$ acts on the product space $M \times N$ freely. And the orbit space $(M \times N) / \Gamma$ is called the dot product of $M$ and $N$ (with respect to $\Gamma$ ) and denoted by $M \cdot N$. In most cases $\Gamma$ will be the group of order two acting on $M$ and $N$ as the covering transformation groups for double covering morphisms in the sequel. $\Gamma$ will not be mentioned in that case, if $\Gamma$ is obvious or if $\Gamma$ need not be specified.

EXAMPLES 3.8a. $\mathrm{SO}(4)=S^{3} \cdot S^{3}, U(n)=T \cdot \mathrm{SU}(n)$ and GI has the only polar $S^{2} \cdot S^{2}$. Here $\Gamma$ for $U(n)$ is the center of $\mathrm{SU}(n)$, a cyclic group of order $n$. 
Proposition 3.9. The dot product for double coverings $M \rightarrow M^{\prime \prime}$ and $N \rightarrow$ $N^{\prime \prime}$ has these properties: (i) $\#_{2} M \leq \#_{2}(M \cdot N)$;

(ii) the fixed point set $F\left(s_{z}, M \cdot N\right)=F\left(s_{o}, M\right) \cdot F\left(s_{p}, N\right) \cup C M \cdot C N$ for every point $z=\pi(o, p)$ of $M \cdot N$; and hence

(iii) $\#_{2}(M \cdot N) \leq \#_{2}\left(F\left(s_{o}, M\right) \cdot F\left(s_{p}, N\right)\right)+\#_{2}(C M \cdot C N)$, where $C M$ and $C N$ are the centrosomes for the point o of $M$ and its pole and for $p$ of $N$ and its pole. Moreover,

(iv) $\frac{1}{2}\left(\#_{2}(M)\right)\left(\#_{2}(N)\right) \leq \#_{2}(M \cdot N) \leq 2\left(\#_{2}\left(M^{\prime \prime}\right)\right)\left(\#_{2}\left(N^{\prime \prime}\right)\right)$.

ProOF. Since the monomorphism: $M \rightarrow M \times N: x \mapsto(x, p)$ induces a monomorphism: $M \rightarrow M \cdot N$, we obtain (i) by 1.4. To prove (ii), we denote by $\pi$ the projection: $M \times N \rightarrow M \cdot N$. Set $z=\pi(o, p)$. Then $s_{z}$ fixes $\pi(x, y)$ in $M \cdot N$ if and only if $\left(s_{o}(x), s_{p}(y)\right)$ is $(x, y)$ or $(\gamma x, \gamma y)$, where the single letter $\gamma$ denotes the double covering transformations on $M$ and $N$. The second case occurs if and only if $(x, y)$ is a point of $C M \times C N$ by 3.4. We have (ii). (iii) is a consequence of (ii). (iv) follows from 3.7 and 1.7 immediately, since the projection: $M \times N \rightarrow M^{\prime \prime} \times N^{\prime \prime}$ is factored through $\pi$.

COROLLARY 3.10 . \# $\#_{2}\left(S^{n} \cdot M\right) \leq \#_{2} M+\#_{2}\left(S^{n-1} \cdot C M\right)$, where $C M$ is a centrosome as in 3.9 .

COROLlary $3.11 . \#_{2} M \leq \#_{2}\left(S^{1} \cdot M\right) \leq \#_{2} M+\#_{2} C M \leq 2 \#_{2} M$.

PROPOSITION 3.12. \#2 $\left(S^{m} \cdot S^{n}\right)=2(n+1)$ if $m \geq n$.

PROOF. We induct on $n$. If $n=0$ or $(m, n)=(1,1)$, we have the equality by 1.6. If $m>1$ and $n \geq 1$, then we have $\#_{2}\left(S^{m} \cdot S^{n}\right)=2+\#_{2}\left(S^{m-1} \cdot S^{n-1}\right)$ by 2.10 .

EXAMPLES 3.13. By $3.12,2.10$ and 3.9 , one obtains $\#_{2}(\mathrm{GI})=1+\#_{2}\left(S^{2} \cdot S^{2}\right)=$ 7. Similarly and by this, $\#_{2} G_{2}=1+\#_{2}(\mathrm{GI})=8$.

LEMMA 3.14. If a compact connected Lie group $G$ has a pole, then (i) \# $2(T \cdot G)$ equals either $\#_{2} G$ or $2 \#_{2} G$, where $T$ is the circle $\mathrm{U}(1)$. (ii) And in the second case the centrosome $C G$ has the 2-number $=\#_{2} G$.

ProOF. We have $\#_{2} G \leq \#_{2}(T \cdot G) \leq 2 \#_{2} G$ by 3.9 and 3.11. But $\#_{2}(T \cdot G)$ is a power of 2 , since $T \cdot G$ is a group too. We have (i). Also (ii) follows from 3.11.

REMARK 3.15. Similarly one obtains an estimate of $\#_{2}(\operatorname{Sp}(1) \cdot G)$; see 8.1.

4. The Euler number $\chi(M)$ and $\#_{2} M$.

THEOREM 4.1. The Euler number $\chi(M) \leq \#_{2} M$ for every compact connected symmetric space $M$.

ProOF. $M$ is a homogeneous space $G / K$; see 2.3 for $G$. We may assume $\chi(M)>0$, since otherwise the inequality is trivial. Thus $G$ is semisimple and the rank $r(K)=r(G)$ by a theorem of Hopf and Samelson [HS]. A maximal torus $A$ of $K$ is that of $G$. Take a vector field $v$ which generates a dense subgroup of $A ; v$ is a member of the Lie algebra of $G$ naturally. Let $V$ be the set of the points of $M$ at which $v$ vanishes. $V$ is clearly the fixed point set of $A$ acting on $M, F(A, M)$. Let $x$ be a point in $V$. Then $x$ is fixed by $A$. Since $A$ is a maximal torus in $G$, $A$ does not fix any nonzero tangent vector at $x ; V$ is a finite set, in particular. 
Also the action of $v$ on the tangent space at $x$ (by the covariant derivative $\nabla v$ ) is nondegenerate (that is, $\operatorname{det}(\nabla v) \neq 0$ at $x$ ). Therefore $\chi(M) \leq \# V=\# F(A, M)$ by Hopf's theorem, where \# denotes the cardinality. The rank condition is known to imply that the symmetries lie in $G$. Therefore the symmetry $s_{x}$ at $x$ belongs to $A$, since it centralizes $A$. Thus $F(A, M)$ is antipodal.

REMARK 4.1a. Actually the equality obtains in the above: $\chi(M)=\# F(A, M)$, since the action of $A$ on the tangent space to $M$ at each point $x$ in $F(A, M)$ is the product of two dimensional rotations, or more precisely the tangent space may be thought of as the direct sum of root spaces with respect to $A$, and hence $\operatorname{det}(\nabla v)>0$ at $x$, that is, each point contributes $+1(=$ the index in Hopf's theorem $)$ to the Euler number.

Proposition 4.2. Assume the rank $r(M)=r(G)$. Then one has $\#_{2} M \geq$ $\chi(M)+2^{r(M)}$ if $M=G / K$ has no pole or if some polar $M^{+}$has the same rank $r(M)$.

Proof. Let $A$ be a maximal torus in $M$. Then $A$ contains an antipodal set $A^{\prime}$ of cardinality $2^{r(M)}$ by 1.6. Thus we may assume $\chi(M)>0$. And we are in the situation of the previous proof of 4.2 , which we will use freely. First we consider the case in which $M$ has no pole. $F(Q(A), M)$ is then antipodal in $M$, where $Q$ is defined with a point $o$ in $A^{\prime}$ (see 2.8), since $Q(A)$ is a maximal torus in $G$. Obviously $A^{\prime}$ is disjoint from $F(Q(A), M)$. We have to show that the union $A^{\prime} \cup F(Q(A), M)$ is antipodal too. Let $p$ be a point of $F(Q(A), M)$. Then $Q(A)$ contains the symmetry $s_{p}$. We have $s_{p}=Q(q)$ for some point $q$ in $A$. Since the symmetry $Q(q)$ is involutive, $s_{q}$ commutes with $s_{o}$ by 3.4. Therefore $s_{p}=Q(q)=s_{q} s_{o}$ commutes with $s_{o}$. Since $s_{p}$ commutes with every member $Q(t)=s_{t} s_{o}$ of $Q(A), s_{p}$ commutes with $s_{t}$ for every point $t$ of $A$. Hence $s_{p}$ fixes every point $t$ of $A$ by 3.4 , since $M$ has no pole. This means $\{p, t\}$ is antipodal. Now we assume that some polar $M^{+}$of $o$ has the same rank $r(M)$ as $M$. Let $A$ be a maximal torus in $K$, the isotropy subgroup of $G$ at $o$. Since maximal tori are conjugate to each other, the orbit $A(p)$ has the maximal dimension $=r(M)$ for some point $p$ in $M^{+}$which is a $K$-orbit (see $[\mathbf{C N}]$ ). Since $s_{o}$ is the identity on $M^{+}, s_{o}$ fixes every point of $A(p)$. This is true for an arbitrary point $x$ in $F(A, M)$, as we are about to prove. On one hand $s_{x}$ is an involutive member of $A$, since $s_{x}$ centralizes $A$. On the other hand an involutive member of $A$ fixes $p$, since the symmetry $s_{p}$ at $p$ stabilizes $A$ and carries every member $b$ into $b^{-1}$ by the assumption. Since $A$ is abelian, it follows that $s_{x}$ fixes every point of $A(p)$. Thus every antipodal set $A^{\prime}$ in $A(p)$ extends to an antipodal set $A^{\prime} \cup F(A, M)$ in $M$, which is of course a disjoint union.

REMARK $4.2 \mathrm{a}$. For example, let $M$ be $\mathrm{CI}(n)^{*}$. Then the inequality holds good for $n=2$ or 4 , but the equality is valid for any other $n$; see 6.5 . The second half applies to $G_{4 m}^{o}\left(\mathbf{R}^{8 m}\right)$ and $G_{4 m}\left(\mathbf{R}^{8 m}\right)$; but the equality does not hold for $G_{4 m}\left(\mathbf{R}^{8 m}\right)$; see 6.1 .

REMARK 4.2b. Picking up one of the assumptions in 4.2 , we can derive a conclusion of some use; if there is a polar $M^{+}$of $o$ having $r\left(M^{+}\right)=r(M)=: r$, then $\#_{2} M>2^{r}$. In fact, $M^{+}$contains a maximal torus $A$ of dimension $r$, which contains an antipodal set $A_{2}$ of $\# A=2^{r}$, and we can add $\{o\}$ to $A_{2}$. For example, $\operatorname{Spin}(n)$ with $n=8 m$ or $8 m+1$ has the polar $G_{4 m}^{o}\left(\mathbf{R}^{n}\right)$. Therefore the 2-rank of $\operatorname{Spin}(n)$ is greater than the rank $4 m$ (see 7.6). 
THEOREM 4.3. One has $\#_{2} M=\chi(M)=1+\Sigma \#_{2} M^{+}$(the equality in 1.9), if $M$ is a hermitian symmetric space of semisimple type.

PROOF. It is known that the Euler number $\chi(M)$ is then positive and all the members of $G$ (see 2.3) are holomorphic transformations of $M$. Hence 4.1 applies. Since the symmetries belong to $G$ as another consequence, all the polars are hermitian; so we can make an induction argument. Since the symmetries are thus homotopic to the identity $1_{M}$, we have $\chi(M)=1+\Sigma \chi\left(M^{+}\right)$, say, by the Lefschetz fixed point theorem in the version of Atiyah and Singer [AS]. In view of 1.9 and the above, we have only to show that every polar ( $\neq$ point) is of semisimple type. Let $p$ be a point in a polar $M^{+}$of $o$. Let $M^{-}$be the orthogonal space (2.13. See [CN] for properties of $\left.M^{-}\right)$. Hence the isotropy subgroup of $G\left(M^{-}\right)$at $p$ coincides with the one at $o$ by 2.9. In particular the center $C$ of $K$, the isotropy subgroup of $G$ at $o$, fixes the point $p$, since $C$ clearly stabilizes the complex submanifold $M^{-}$. Therefore $C$ acts trivially on $M^{+}$, since $M^{+}$is a $K$-orbit (see [CN]). We conclude that $M^{+}$is of semisimple type, now that $M^{+}$is an orbit of the semisimple part of $K$.

COROLLARY 4.4. If a compact connected hermitian symmetric space $M$ is a subspace of another compact connected hermitian symmetric space $N$, then one has $\chi(M) \leq \chi(N)$.

5. Classical groups. We will determine the 2-numbers $\#_{2}$ for individual spaces. In this section, we take a look at classical groups. Some of the results (for $\mathrm{U}(n), \mathrm{SO}(n)$ and $\mathrm{Sp}(n))$ are found in [BSe].

Notation 5.1. If $M$ is a group, $r_{2}(M)$ denotes the 2-rank of $M$ (see 1.3).

PROPOSITION 5.2. Let $\mathrm{U}(n) / \mathbf{Z} \mu$ by the quotient group of the unitary group $\mathrm{U}(n)$ by the cyclic normal subgroup $\mathbf{Z} \mu$ of order $\mu$. Then $r_{2}(\mathrm{U}(n) / \mathbf{Z} \mu)$ is $n+1$ if $\mu$ $i s$ even and $n=2$ or 4 ; and it is $=n$ otherwise.

Proof. We write $M$ for $\mathrm{U}(n)$ and $M^{\prime \prime}$ for $\mathrm{U}(n) / \mathbf{Z} \mu$ in the proof, which will take a few lemmas. Our method is to use linear algebra. Let $\pi$ denote the projection: $M \rightarrow M^{\prime \prime}$. Let $B$ be the inverse image $\pi^{-1}\left(A_{2}\right)$ of a maximal antipodal subgroup $A_{2}$ in $M^{\prime \prime}$ under $\pi$. We identify the circle group $\mathrm{U}(1)=\{z \in C:|z|=1\}$ with the center of $\mathrm{U}(n)$. We fix a primitive $2 \mu$ th root of 1 and call it $\theta \in \mathrm{U}(1)$.

LEMMA 5.3. $\theta B=B$.

ProOF. First note that $\theta^{2} B=B$ obviously. If $a, b$ are members of $B$, then

$$
\pi(\theta a)^{2}=\pi(\theta)^{2} \pi(a)^{2}=1
$$

by 1.3 and

$$
\pi(\theta a) \pi(b)=\pi(\theta) \pi(a) \pi(b)=\pi(b) \pi(\theta) \pi(a)=\pi(b) \pi(\theta a)
$$

by 1.3 , since $\pi(\theta)$ lies in the center of $M^{\prime \prime}$. Hence $\theta a \in B$ by the maximality of $A_{2}$.

LEMMA 5.4. If $B$ is abelian, then \# $A_{2}=2^{n}$, and, more precisely, $A_{2}$ is conjugate in $\mathrm{U}(n)$ with $\pi\{1, \theta\} \Delta$, where $\Delta:=\Delta(\mathrm{O}(n))$ is the subgroup of $\mathrm{O}(n) \subset \mathrm{U}(n)$ consisting of the diagonal matrices.

Proof. $B$ is then simultaneously diagonalizable. Let $c \in B$. Since $c^{2}=\phi$ for some $\phi$ in $\mathbf{Z} \mu$, the projection $\pi(c)=\pi(b)$ for some $b$ in $B$ satisfying $b=I$ or $b=\theta I$ 
for some $I$ in $\Delta$. Thus $A_{2}$ is contained in $\pi\{1, \theta\} \Delta$, or so we may assume. Here we have the equality actually by 5.3 , since $A_{2}$ is maximal. $\# \Delta=2^{n}=\# \pi\{1, \theta\} \Delta$, since the $\mu$ th power of $\theta$ is -1 . The rest is now obvious.

LEMMA 5.5. If $a b \neq b a$ for some $a, b$ in $B$, then $a b=-b a$.

ProOF. $a b=\phi b a$ for some $\phi$ in $\mathbf{Z} \mu$, since $A_{2}$ is abelian. Hence $b^{2}=\left(\phi a^{-1} b a\right)^{2}=$ $\phi^{2} b^{2}$ since $b^{2}$ lies in the center (which contains the kernel of $\pi$ ). Hence $\phi^{2}=1$.

LEMMA 5.6. If $a, b, c \in B$ and $a b=-b a$, then some one of $c, a c, b c$, and $a b c$ commutes with both $a$ and $b$.

PROOF. Direct computations show this, since $a c= \pm c a$ and $b c= \pm c b$ by 5.5 .

LEMMA 5.7. If $a b=-b a$ for $a$ and $b$ in $B$, then (i) the trace $\operatorname{Tr}(a)=\operatorname{Tr}(b)=0$, (ii) $n=2 n^{\prime}$ is even, (iii) $a$ and $b$ are conjugate in $\mathrm{U}(n)$ with scalar multiples of $I_{n^{\prime}}$ and (iv) $\mu$ is also even.

ProOF. From $a=-b a b^{-1}$, we have $\operatorname{Tr}(a)=-\operatorname{Tr}\left(b a b^{-1}\right)=-\operatorname{Tr}(a)$, and $\operatorname{Tr}(a)=0$. Similarly for $b$. In the notation of the proof of 5.4 , this means $\operatorname{Tr}(I)=0$. Thus $n$ is even and $I$ is conjugate with $I_{n^{\prime}}, n^{\prime}=n / 2$. For (iv), notice that $-1=\phi$ in the proof of 5.5. Hence -1 is a $\mu$ th root of 1 .

At this point, we explain where we are in the proof of 5.2. If $B$ is abelian for a great $A_{2}$, then we have $\#_{2} M^{\prime \prime}=2^{n}$ or $r_{2}=n$ by 5.4. This is the case when $n$ or $\mu$ is odd, by 5.7 . We have $r_{2}\left(M^{\prime \prime}\right) \geq n$ always by 2.7. If $B$ is not abelian for any great $A_{2}$, we assert that $\{a, b\}$ in 5.5 generates a group which is isomorphic with the dihedral group $D[4]$ in 1.5 and that $A_{2}$ is contained in a group $\cong \pi\{1, \theta\} D[4] \otimes \mathrm{U}\left(n^{\prime}\right)$. It follows that $r_{2} M \leq 2+r_{2}\left(\mathrm{U}\left(n^{\prime}\right) / \mathrm{Z} \mu\right)$. This will be enough.

LEMMA 5.8. If $B$ is not abelian, then $A_{2}$ is conjugate with a subgroup of $\pi\{1, \theta\} D[4] \otimes \mathrm{U}\left(n^{\prime}\right)$.

ProOF. If two members $a, b$ of $B$ do not commute with each other, then $a, b$ are conjugate with $I_{n^{\prime}}$ by 5.7 , or so we may assume by 5.3 . This means $a$ and $b$ lie in the polar $G_{n^{\prime}}\left(C^{n}\right)$ of 1 in $\mathrm{U}(n)$. Since $a b=-b a$, the point $b$ lies in the centrosome $C(a,-a) \cong \mathrm{U}\left(n^{\prime}\right)$ in this polar by 1.3 and 3.4. Since $\mathrm{U}\left(n^{\prime}\right)$ is connected, the pair $(a, b)$ is conjugate with the particular pair of $I_{n^{\prime}}=I_{1} \otimes 1_{n^{\prime}}$ and $K_{n^{\prime}}=K_{1} \otimes 1_{n^{\prime}}$. Now 5.6 gives the lemma, in which $\theta$ may be omitted; $\{1, \theta\} D[4] \otimes \mathrm{U}\left(n^{\prime}\right)=\left(D[4] \otimes \mathrm{U}\left(n^{\prime}\right)\right)=\left(Q[8] \otimes \mathrm{U}\left(n^{\prime}\right)\right)$.

COROLlaRY 5.9. If $B$ is not abelian for any great $A_{2}$, then $r_{2}\left(M^{\prime \prime}\right) \leq 2+$ $r_{2}\left(\mathrm{U}\left(n^{\prime}\right) / \mathbf{Z} \mu\right)$.

We will conclude the proof of 5.2. We have only to consider the case where both $n$ and $\mu$ are even and $B$ is not abelian. Then, in case $n=2$, the right-hand side $2+r_{2}\left(\mathrm{U}\left(n^{\prime}\right) / \mathrm{Z} \mu\right)$ in 5.9 equals $2+r_{2}(\mathrm{U}(1))=3$, while $\pi\{1, \theta\} D[4]$ has the 2-rank=3. If $n=4,2+r_{2}\left(\mathrm{U}\left(n^{\prime}\right) / Z \mu\right)=5$ and $\pi\{1, \theta\} D[4] \otimes D[4]$ has the 2-rank 5 . For $n>4$, an easy induction on $n$ completes the proof.

PROPOSITION 5.10. $r_{2}(\mathrm{SU}(n) / \mathrm{Z} \mu)$ is $n+1$ for $(n, \mu)=(4,2), n$ for $(n, \mu)=$ $(2,2)$ or $(4,4)$ and $n-1=r_{2}(\mathrm{SU}(n) / \mathrm{Z} \mu)$ for the other cases.

The proof is similar to that of 5.2. A difference is, of course, that $\operatorname{det}(b)=1$ for $b$ in $\mathrm{SU}(n)$. Hence $\mu$ divides $n$ and $I_{p}$ belongs to $\mathrm{SU}(n)$ if and only if $p$ is even, while 
$\theta I_{p}$ is in $\mathrm{SU}(n)$ if and only if $1=\operatorname{det}\left(\theta I_{p}\right)=\theta^{n}(-1)^{p}$. We have $r_{2}\left(\mathrm{SU}(2)^{*}\right)=2$ by 2.11 , since $\mathrm{SU}(2)^{*}=\left(S^{3}\right)^{*}$.

REMARK 5.11. The next Propositions 5.12 through 5.14 are concerned with the other classical groups and their adjoint groups and proven with the same method as 5.2 basically. In each proof we will point out the difference and the changes we have to make. Here we explain some common features and common notations. For the group $M:=\mathrm{O}(n), \mathrm{SO}(n)$ or $\mathrm{Sp}(n)$, its 2-rank is easy to find; an antipodal subgroup is diagonalizable (obviously at least if $M$ is $\mathrm{O}(n)$ or $\mathrm{SO}(n)$ ). For $M^{\prime \prime}:=$ $\mathrm{O}(n)^{*}=\mathrm{O}(n) /\{ \pm 1\}$ for $n \geq 1, \mathrm{SO}(n)^{*}$ for $n$ even and $>2$, or $\mathrm{Sp}(n)^{*}$, we denote by $B$ the inverse image of a given great antipodal subgroup $A_{2}$ of $M^{\prime \prime}$ under the projection $\pi: M \rightarrow M^{\prime \prime}$, which is a 2-to-1 morphism. Every member of $B$ thus satisfies $x^{2}= \pm 1$. Case $1.1^{\circ}$ : if $B$ is abelian and all the members are involutive, then $B$ is a maximal antipodal subgroup of $M$, and hence we obtain the 2-rank $r_{2}\left(M^{\prime \prime}\right)=r_{2}(M)-1$, since the projection of a great antipodal subgroup is antipodal. This is normally the case. Case $1.2^{\circ}$ : if $B$ is not antipodal but abelian, then $B$ meets the centrosome $C$ at a point, say $c$. Hence $B$ is a subgroup of $F(\operatorname{ad}(c), M)$ (which will be a unitary group). Case $2^{\circ}$ : if $B$ is not abelian, then $B$ contains members $a, b$ satisfying $a b=-b a$ by 5.5 . These $a$ and $b$ generate a dihedral group $D[4]$ (Case $2.1^{\circ}$ ) or a quaternion group $Q[8]$ (Case $2.2^{\circ}$ ), according as one of $a, b$ and $a b$ is involutive or not; the distinction is necessary because of the lack of $\theta$ used in the proof of 5.2. Hence $B$ is a subgroup of $D[4] \cdot Z$ or $Q[8] \cdot Z$ accordingly by 5.6 , where $Z$ is the centralizer of the dihedral or quaternion group in $M$. We will then induct on $n$ in dealing with the other case for $B$. Then a minor complexity occurs, however, because in the reduction stage of the induction different types of groups come into the scene (for instance, $Z=\mathrm{O}\left(n^{\prime}\right)$ or $\mathrm{Sp}\left(n^{\prime \prime}\right)$ into the proof for $\left.\mathrm{SO}(n)^{*}\right)$. Thus, theoretically, all those groups must be considered simultaneously with induction of a larger scale, but we prefer a simpler presentation which might look like a vicious circle (and which is not). In determining $D[4]$ and $Q[8]$, we use the fact: the relation $a b=-b a$ implies that the symmetries at $a$ and $b$ commute with each other and hence the point $a$ lies in the centrosome for $(b,-b)$ on any connected space in $M$ which carries these three points by 3.4 .

PROPOSITION 5.12. One has $r_{2}(\mathrm{SO}(n))=n-1$, while $r_{2}\left(\mathrm{SO}(n)^{*}\right)$ is 4 for $n=4$ and $n-2$ for $n$ even $>4$. (Of course, $\mathrm{SO}(n)^{*}$ is $\mathrm{SO}(n)$ for $n$ odd and $\mathrm{SO}(n) /\{ \pm 1\}$ if $n=2 n^{\prime}$ is even $>2$.)

ProOF. First note $\mathrm{SO}(4)^{*}=\mathrm{SU}(2)^{*} \times \mathrm{SU}(2)^{*}$ has 2 -rank 4 by 5.10 . We use the notations in 5.11 with $M=\mathrm{SO}(n)$ and $M^{\prime \prime}=\mathrm{SO}(n)^{*}$. In Case $1.2^{\circ}, B$ is contained in $\mathrm{U}\left(n^{\prime}\right)=F(\operatorname{ad}(c), M)$. Hence the 2-rank of $A_{2}$ is $r_{2}\left(\mathrm{U}\left(n^{\prime}\right) / \mathbf{Z}_{2}\right)<n-1$ by 5.2. In Case $2.1^{\circ}, B$ contains $D[4]$, which has generators $a, b$ contained in the polar $G_{n^{\prime}}\left(\mathbf{R}^{n}\right), n=2 n^{\prime}$ even, since $\operatorname{Tr}(a)=0$ by (i) of 5.7. Write $J$ for $a b$. Then an involutive member $x$ of $M$ satisfies $J x=-x J$ if and only if $x=I_{n^{\prime}} y$ for some $y$ in $\mathrm{U}\left(n^{\prime}\right)$ defined by $J$. The condition $x^{2}=1$ reads $y \in \mathrm{UI}\left(n^{\prime}\right)$ which is connected. Thus we may assume $a=I_{n^{\prime}}$ (hence $n^{\prime}$ is even). Thus $B$ is a subgroup of $D[4] \otimes O\left(n^{\prime}\right)=D[4] \cdot \mathrm{O}\left(n^{\prime}\right)$. Hence $r_{2}\left(M^{\prime \prime}\right) \leq 2+r_{2}\left(\mathrm{O}\left(n^{\prime}\right)^{*}\right)$. Therefore one has $r_{2}\left(A_{2}\right) \leq n-2$ for $n>4$ by 5.13 (or by 5.2 and $\mathrm{O}\left(n^{\prime}\right) \subset \mathrm{U}\left(n^{\prime}\right)$ ). In Case $2.2^{\circ}$, the generators of $Q[8]$ are contained in the centrosome $S=2 \times \operatorname{DIII}\left(n^{\prime}\right)$. Since an automorphism of $M$ exchanges these two components, we may assume 
that $J=J_{1} \otimes 1_{n^{\prime}}$ is one of the generators. The number $n^{\prime}=2 n^{\prime \prime}$ is even, by $x J=-J x \Leftrightarrow K x \in \mathrm{U}\left(n^{\prime}\right)$ where $K=K_{1} \otimes 1_{n^{\prime}}$; note that $\operatorname{det}(K)=(-1)^{n^{\prime}}$. It is not hard to see that they lie in a single component. Since the centrosome in $\operatorname{DIII}\left(n^{\prime}\right)$ is $\operatorname{AII}\left(n^{\prime \prime}\right)$ which is connected, $Q[8]$ is also unique up to automorphisms. Thus we may assume that $Q[8]$ is generated by $J$ and $I_{1} \otimes J_{1} \otimes 1_{n^{\prime \prime}}$. Hence its centralizer $Z$ is isomorphic with $\operatorname{Sp}\left(n^{\prime \prime}\right)$. Thus $B \subset Q[8] \otimes \operatorname{Sp}\left(n^{\prime \prime}\right)$. Since $\operatorname{Sp}\left(n^{\prime \prime}\right)$ is a subgroup of $\mathrm{SU}\left(n^{\prime}\right)$, we obtain $r_{2}\left(A_{2}\right)<n-1$ for $n=6$ or $n>8$ by 5.10 and even for $n=8$ by $r_{2}\left(\operatorname{Sp}(2)^{*}\right)=r_{2}(\mathrm{SO}(5))=4$.

PROPOSITION 5.13. $r_{2}(\mathrm{O}(n))=n . r_{2}\left(\mathrm{O}(n)^{*}\right)$ is $n$ if $n$ is 2 or 4, while it is $n-1$ otherwise, where $\mathrm{O}(n)^{*}:=\mathrm{O}(n) /\{ \pm 1\}$.

PrOOF. An antipodal subgroup of $\mathrm{O}(n)$ is diagonalizable, and hence the 2-rank of $\mathrm{O}(n)$ is $n$. Note that $\mathrm{O}(n)^{*} \cong \mathrm{SO}(n)$ if $n$ is odd. We may assume that $B$ is not contained in $\mathrm{SO}(n)$. Then the Cases $1.2^{\circ}$ and $2.2^{\circ}$ are excluded. The Case $2.1^{\circ}$ is similar to 5.13 except that $D[4] \otimes \mathrm{O}\left(n^{\prime}\right)=D[4]$ for $n=2$ and we have the 2-rank $=2$ of $\mathrm{O}(2)^{*}$. The assumption implies that $n^{\prime}$ is odd. For $n>4, B$ does not give the 2 -rank in this case.

PROPOSITION 5.14. One has $r_{2}(\operatorname{Sp}(n))=n$, while $r_{2}\left(\operatorname{Sp}(n)^{*}\right)$ is $n+2$ for $n=2$ or 4 and $n+1$ otherwise. Thus $r_{2}\left(\operatorname{Sp}(n)^{*}\right)=r_{2}\left(\mathrm{U}(n) / Z_{2}\right)+1$ for every $n$.

PROOF. First we will show that an antipodal group $A_{2}$ in $\operatorname{Sp}(n)$ is "diagonalizable" (or rather contained in a maximal torus). Since $\operatorname{Sp}(n)$ is 1-connected, $A_{2}$ is contained in the orthogonal space $\operatorname{Sp}(d) \times \operatorname{Sp}(n-d)$ to a polar $G_{d}\left(H^{n}\right)$ which $A_{2}$ meets, by 2.13. Therefore one has

$$
r_{2}(\operatorname{Sp}(n))=r_{2}(\operatorname{Sp}(d) \times \operatorname{Sp}(n-d))=r_{2}(\operatorname{Sp}(d))+r_{2}(\operatorname{Sp}(n-d))=d+(n-d)
$$

by induction on $n$ beginning with $r_{2}(\mathrm{Sp}(1))=r_{2}(\mathrm{SU}(2))=1$. The 2-ranks of $\mathrm{Sp}(1)^{*}=\mathrm{SU}(2)^{*}$ and $\mathrm{Sp}(2)^{*}=\mathrm{SO}(5)$ are known to be 2 and 4 respectively. Referring to 5.11 , we begin with $2.2^{\circ}$; the Case $1.1^{\circ}$ of $1.2^{\circ}$ cannot occur as we will show shortly. Case $2.2^{\circ}: B$ contains $Q[8]$ generated by two members $a$ and $b$ of the centrosome $\mathrm{CI}(n)$ by (i) and (ii) of 5.7. Since the centrosome $\mathrm{UI}(n)$ of $\mathrm{CI}(n)$ is connected, we may assume $a=J_{1} \otimes 1_{n}$ and $b=i \mathrm{I}_{n}$. Thus $B$ is contained in $Q[8] \otimes \mathrm{O}(n)$. Therefore $r_{2}\left(A_{2}\right) \leq 2+r_{2}\left(\mathrm{O}(n)^{*}\right)$; and hence, $r_{2}\left(A_{2}\right) \leq 2+(n-1)=n+1$ by 5.13 if $n \neq 2$ or 4 . The same 6.13 also gives the correct 2 -rank of $\operatorname{Sp}(4)^{*}$. Let $\Delta=\Delta(n)$ denote the subgroup of the diagonal matrices in $\mathrm{O}(n)$. Then a group $B$ in the Case $1.1^{\circ}$ or $1.2^{\circ}$ is necessarily a subgroup of $Q[8] \otimes \Delta$ (or a conjugate thereof), since $Q[8] \otimes \Delta$ contains every diagonal and involutive member of $\operatorname{Sp}(n)$ and every diagonal member of $\mathrm{U}(n)=F\left(\operatorname{ad}\left(J_{1} \otimes 1_{n}\right), \operatorname{Sp}(n)\right)$ which satisfies $x^{2}= \pm 1$. The 2-rank of $\pi(Q[8] \otimes \Delta)$ is $n+1$. Case 2.1 $1^{\circ}$ : the polar $G_{n^{\prime}}\left(H^{n}\right)$ contains two generators $a$ and $b$ of $D[4]$ by (i) and (ii) of $5.7 ; n=2 n^{\prime}$. Since the centrosome $\operatorname{Sp}\left(n^{\prime}\right)$ in it is connected, we may assume $a=1_{2} \otimes I_{1} \otimes 1_{n^{\prime}}$ and $b=1_{2} \otimes K_{1} \otimes 1_{n^{\prime}}$. Hence $B$ is contained in $D[4] \otimes \operatorname{Sp}\left(n^{\prime}\right)$; here this $\operatorname{Sp}\left(n^{\prime}\right)$ is the diagonal subgroup in $\operatorname{Sp}\left(n^{\prime}\right) \times \mathrm{Sp}\left(n^{\prime}\right) \subset \mathrm{SU}(2 n)$ and we have identified $\operatorname{Sp}(n)$ with $F(\kappa \circ \operatorname{ad}(J), \mathrm{SU}(2 n))$. Thus $r_{2}\left(A_{2}\right) \leq 2+r_{2}\left(\operatorname{Sp}\left(n^{\prime}\right)^{*}\right)$ and, inductively, we obtain the 2 -rank $\leq 2+\left(n^{\prime}+1\right) \leq n+1$ for $n \geq 6$.

REMARK 5.15. We make some comments on the proofs of Propositions 5.12 through 5.14 for a later reference. Let $B$ denote the inverse image of a maximal 
antipodal subgroup of $\operatorname{Sp}(n)^{*}$ under the projection $\pi: \operatorname{Sp}(n) \rightarrow \operatorname{Sp}(n)^{*}$. We will describe $B$ up to conjugacy in $\operatorname{Sp}(n)$. We have seen in the proof of 5.14 that $B$ is a subgroup of either $Q[8] \otimes \mathrm{O}(n)$ or $D[4] \otimes \mathrm{Sp}(m), n=2 m$. $B$ for $n=2$ is $Q[8] \otimes D[4]$. $B$ for $n=4$ is one of $Q[8] \otimes Q[8] \otimes Q[8], D[4] \otimes Q[8] \otimes Q[8], Q[8] \otimes D[4] \otimes D[4]$ and $Q[8] \otimes \Delta$, where $\Delta:=\Delta(n)=\Delta(\mathrm{O}(n))$ is the subgroup of $\mathrm{O}(n)$ consisting of the diagonal matrices.

\section{Classical spaces.}

Proposition 6.1. $\#_{2} G_{p}(n)=\left(\begin{array}{l}n \\ p\end{array}\right)$, the binomial coefficient. Moreover the great antipodal sets are congruent with each other under the automorphism group.

PROOF. A point $x$ in $G_{p}(n)$ is a $p$-dimensional subspace $[x]$ of the metric cartesian $n$-space $V$ over $\mathbf{R}, \mathbf{C}$ or $\mathbf{H}$. We work on the real one for simplicity; the automorphism group of $V$ is $\mathrm{O}(n)$. Let $P_{x}$ be the orthogonal projection of $V$ onto $[x]$. Then the natural action of $2 P_{x}-1_{n}$ on $G_{p}(n)$ is the symmetry $s_{x}$; we denote it by $s_{[x]}$. The map $f: G_{p}(n) \rightarrow \mathrm{O}(n): x \mapsto s_{[x]}$ is a monomorphism. Since $[x]$ is an eigenspace of $s_{[x]}$ and the orthogonal complement is the other eigenspace (in fact $s_{[x]}$ is conjugate with $-I_{p}$ in $\left.\mathrm{O}(n)\right)$, it is easy to see that $s_{x}$ fixes a point $y$ in $G_{p}(n)$ if and only if $s_{[x]}$ commutes with $s_{[y]}$. The desired 2-number is then the number of matrices in $\Delta$ the diagonals in $\mathrm{O}(n)$, whose trace is $2 p-n$. The second half is also clear now.

\section{Proposition 6.2. $\#_{2}(\mathrm{CI}(n))=2^{n}$ and $\#_{2}(\mathrm{DIII}(n))=2^{n-1}$.}

ProOF. These are hermitian and Theorem 4.3 applies. The Euler numbers are the sums of those of the fixed point sets of the symmetries.

REMARK 6.2a. We will prove the Propositions 6.4, 6.5 and 6.6 simultaneously by induction arguments because of the complicated situation which is similar to the one with classical groups as mentioned in 5.11. The Grassmann manifolds are polars in the classical groups $\mathrm{U}(n), \mathrm{O}(n)$ and $\operatorname{Sp}(n)$, while the space $\operatorname{DIII}(n)$ (or rather its 2 copies) and $\mathrm{CI}(n)$ appear as the centrosomes in $\mathrm{O}(n)$ and $\operatorname{Sp}(n)$. $G_{m}(2 m), \operatorname{DIII}(n)$ and $\mathrm{CI}(n)$ are the double covering spaces of their bottom spaces. The projections are the restrictions of those, denoted commonly by $\pi: M \rightarrow M^{\prime \prime}$, of the ambient classical groups $M$ onto the quotient groups $M^{\prime \prime}$ by the groups of order 2 in their centers. As in 5.11, we denote by $B$ the inverse image of a maximal antipodal subgroup of $M^{\prime \prime}$ under $\pi$ or a conjugate subgroup to it in $M$ which we choose in the conjugate class. In case $M=\operatorname{Sp}(n), B$ is contained in $D[4] \otimes \operatorname{Sp}(n / 2)$ or $Q[8] \otimes \mathrm{O}(n)$ (see 5.15 ; it was the main step in the proof of 5.14 to establish this fact). If $M=\mathrm{O}(n), B$ is either $\Delta$ or a subgroup of $\mathrm{U}(n / 2)$, $D[4] \otimes \mathrm{O}(n / 2)$ or $Q[8] \otimes \mathrm{Sp}(n / 4)$ similarly, where $\Delta:=\Delta(\mathrm{O}(n))$ is the subgroup of $\mathrm{O}(n)$ consisting of the diagonal matrices. In case $M=\mathrm{U}(n), B$ is $\{1, i\} \Delta$ or a subgroup of $\{1, i\} D[4] \otimes \mathrm{U}(n / 2)$, where $i$ is the imaginary unit, $i^{2}=-1$, and the presence of $\{1, i\}$ corresponds to 5.3 ; note $\{1, i\} D[4]=\{1, i\} Q[8]$ in $\mathrm{U}(n)$. Since the points of $G_{m}(2 m)$ are characterized in $M$ by the conditions $\operatorname{Tr}(x)=0$ and $x^{2}=1$ and those of the centrosome by $x^{2}=-1$, the next lemma will be useful in proving the propositions.

LEMMA 6.3. Among the points of $Q[8] \otimes D[4]$, those which satisfy $x^{2}=1$ are 12 in number and there are 20 points which satisfy $x^{2}=-1$; among the former points 
exactly 10 points satisfy $\operatorname{Tr}(x)=0$ too. Similarly, among the points of $Q[8] \otimes Q[8]$ and $D[4] \otimes D[4]$, those which satisfy $x^{2}=1$ are 20 in number and there are 12 points which satisfy $x^{2}=-1$; among the former points exactly 18 points satisfy $\operatorname{Tr}(x)=0$ too.

PROOF. Straightforward by $\operatorname{Tr}(x \otimes y)=\operatorname{Tr}(x) \operatorname{Tr}(y)$ and $(x \otimes y)^{2}=x^{2} \otimes y^{2}$.

Proposition 6.4. \# $\#_{2}\left(G_{m}(2 m)^{*}\right)=\left(\begin{array}{c}2 m \\ m\end{array}\right) / 2$ with the following exceptions. $\#_{2}\left(G_{2}\left(\mathbf{R}^{4}\right)^{*}\right)=9 ; \#_{2}\left(G_{m}\left(\mathbf{C}^{2 m}\right)^{*}\right)$ is 3 for $m=1,15$ for $m=2,12$ for $m=3$ and 63 for $m=4 ; \#_{2}\left(G_{m}\left(\mathbf{H}^{2 m}\right)^{*}\right)$ is 5 for $m=1,27$ for $m=2,20$ for $m=3$ and 135 for $m=4$.

PROPOSITION 6.5. $\#_{2}\left(\mathrm{CI}(n)^{*}\right)$ is $2^{n}+2^{n-1}$ except that it is 10 for $n=2$ and 36 for $n=4$.

Proposition 6.6. $\#_{2}\left(\operatorname{DIII}(n)^{*}\right), n$ even, is $2^{n-2}$ except that it is 3 for $n=2$, 28 for $n=4$ and 120 for $n=8$. (If $n$ is odd, $\operatorname{DIII}(n)^{*}$ coincides with $\operatorname{DIII}(n):=$ $\mathrm{SO}(2 n) / \mathrm{U}(n)$.

Now we begin in the proof of the three propositions. We already know the 2numbers of $G_{1}\left(C^{2}\right)^{*}=\mathrm{CI}(1)^{*}=\operatorname{DIII}(2)^{*}=S^{2 *}, G_{1}\left(H^{2}\right)^{*}=\left(S^{4}\right)^{*}, G_{2}\left(\mathbf{R}^{4}\right)^{*}=$ $\left(S^{2}\right)^{*} \times\left(S^{2}\right)^{*}, G_{2}\left(C^{4}\right)^{*}=G_{2}\left(\mathbf{R}^{6}\right), \mathrm{CI}(2)^{*}=G_{2}\left(\mathbf{R}^{5}\right)$, and $\operatorname{DIII}(4)^{*}=G_{2}\left(\mathbf{R}^{8}\right)$; they are $3,5,9,15,10$, and 28 respectively. In the notations of $6.2 \mathrm{a}$, we take $M=\operatorname{Sp}(n)$ first. Then $B$ is a subgroup of $Q[8] \otimes \mathrm{O}(n)$ or $D[4] \otimes \mathrm{Sp}(n / 2)$. If $B=Q[8] \otimes \Delta$, then $B$ meets $\mathrm{CI}(n)$ at $3\left(2^{n}\right)$ points and $G_{m}\left(\mathbf{H}^{2 m}\right)$ at $\#_{2}\left(G_{m}\left(\mathbf{H}^{2 m}\right)\right)$ points (if $n=2 m$ is even). Hence $\#_{2}\left(\mathrm{CI}(n)^{*}\right) \geq 2^{n}+2^{n-1}$ and $\#_{2}\left(G_{m}\left(\mathbf{H}^{2 m}\right)^{*}\right) \geq \frac{1}{2} \#_{2}\left(G_{m}\left(\mathbf{H}^{2 m}\right)\right)$; notice that these are the stated regular values of the 2-numbers of these spaces. We have the equality for $\mathrm{CI}(n)^{*}$ if $n$ is odd, in particular (since there is no other choice of $B$ then). If $B$ is not $Q[8] \otimes \Delta, B$ is contained in one of $Q[8] \otimes \mathrm{O}(2 m), Q[8] \otimes \mathrm{U}(m)$, $Q[8] \otimes D[4] \otimes \mathrm{O}(m), Q[8] \otimes Q[8] \otimes \mathrm{Sp}(m / 2)$ and $D[4] \otimes \mathrm{Sp}(m)$; therefore the order $\# B$ is less than or equal to $2^{4+m}$. Thus we have the stated value $\#_{2}\left(G_{m}\left(\mathbf{H}^{2 m}\right)^{*}\right)=$ $\frac{1}{2} \#_{2}\left(G_{m}\left(\mathbf{H}^{2 m}\right)\right)$ for $m>6$, since the binomial coefficient $\#_{2}\left(G_{m}\left(\mathbf{H}^{2 m}\right)\right)$ is greater than $2^{4+m}$ for $m>6$. This proves 6.4 for $m>6$ by 3.7 and the obvious fact: $G_{m}\left(\mathbf{R}^{2 m}\right)^{*} \subset G_{m}\left(\mathbf{C}^{2 m}\right)^{*} \subset G_{m}\left(\mathbf{H}^{2 m}\right)^{*}$.

We now work on the cases $m=2,3,4,5$ and 6 for $G_{m}\left(\mathbf{H}^{2 m}\right)^{*}$. For $M=\operatorname{Sp}(4)$, the case $B=Q[8] \otimes Q[8] \otimes Q[8]$ gives the correct values for $\mathrm{CI}(4)^{*}$ and $G_{2}\left(H^{4}\right)^{*}$ by 6.3 , because the other 3 cases do not meet $\mathrm{CI}(4)$ and $G_{2}\left(\mathbf{H}^{4}\right)$ at more points as is easily seen. One obtains the correct values for $m=3$ and 4 are with $B=$ $Q[8] \otimes D[4] \otimes \Delta(3)$ and $Q[8] \otimes Q[8] \otimes D[4] \otimes D[4]$ respectively. Similarly one verifies the cases $m=5$ and 6 are regular. Actually one obtains the results for $\mathrm{CI}(n)^{*}$ with $n=2 m=4$ and 6 simultaneously by 6.3 or in the spirit thereof; in particular one has the formula $\#_{2} \mathrm{Sp}(4)^{*}=1+\#_{2} \mathrm{CI}(4)^{*}+\#_{2} G_{2}\left(\mathbf{H}^{4}\right)^{*}$. As to the regular values, the estimate using the same number $2^{4+m}$ is even better for $\mathrm{CI}(n)^{*}$.

One obtains the stated values for $G_{m}\left(\mathbf{C}^{2 m}\right)^{*}, m=3$ and 4 , by $B=\{1, i\} D[4] \otimes$ $\Delta(3)$ and $\{1, i\} D[4] \otimes D[4] \otimes D[4]$ in $\mathrm{U}(2 m)$. Those for $G_{m}\left(\mathbf{R}^{2 m}\right)^{*}, m=3$ and 4 , are obtained by $B=\Delta(m)$ in $\mathrm{O}(2 m)$.

Finally we look at $\operatorname{DIII}(n)^{*}, n=2 m$. Its 2-number is greater than or equal to $2^{n-2}$ by 6.2 and 3.7 . This case is slightly complicated, because the centrosome for $(1,-1)$ in $\mathrm{SO}(4 m)$ is not connected but the disjoint union of 2 copies of $\operatorname{DIII}(n)$. The outer automorphism $\operatorname{ad}\left(I_{k}\right), k$ odd, carries one into the other. Hence 
$B:=\{1, i\} \Delta(2 m) \subset \mathrm{U}(2 m) \subset \mathrm{SO}(4 m)$ gives the regular value $2^{n-2}$. It is easy to see that $B$ meets a single component $\operatorname{DIII}(n)$ if $B$ has $Q[8]$ among its factors. We have only to consider the cases $m=3$ or 4 by the estimate which is similar to the one for $G_{m}\left(\mathbf{H}^{2 m}\right)^{*}$. The case $m=3$ is regular. In case $m=4, B=Q[8] \otimes Q[8] \otimes D[4] \otimes D[4]$ gives the correct value. And the Propositions 6.4 through 6.6 have been proven.

REMARK 6.7. The above result together with 1.4 preclude the possibility of an inclusion $G_{2}\left(C^{4}\right)^{*} \subset G_{3}\left(C^{6}\right)^{*}$ and $\operatorname{DIII}(4)^{*} \subset \operatorname{DIII}(6)^{*}$.

\section{Other classical spaces.}

Proposition 7.1. Let $\mathrm{UI}(n)$ denote $\mathrm{U}(n) / \mathrm{O}(n)$. Then $\#_{2}(\mathrm{UI}(n) / \mathrm{Z} \mu)$ is 6 if $n=2$ and $\mu$ is even; it is 20 if $n=4$ and $\mu$ is even; and it is $2^{n}$ otherwise.

Proof. We write $M$ for $\mathrm{UI}(n):=\mathrm{U}(n) / \mathrm{O}(n)$ and $M^{\prime \prime}$ for $\mathrm{UI}(n) / \mathbf{Z} \mu . M$ has rank $n$ and a subspace of $\mathrm{U}(n)$ characterized as $F(\kappa \circ \iota, \mathrm{U}(n))$. Thus we have $2^{n} \leq \#_{2}\left(M^{\prime \prime}\right) \leq \#_{2}(\mathrm{U}(n) / \mathrm{Z} \mu)=2^{n}$ and hence the proposition, by 2.7 and 5.2 , unless $\mu$ is even and $n=2$ or 4 . $M$ is the inverse image of $M^{\prime \prime}$ under the projection $\pi$ of $\mathrm{U}(n)$ onto $\mathrm{U}(n) / \mathrm{Z} \mu$. Let $B$ be the inverse image of a maximal antipodal group in $\mathrm{U}(n) / \mathrm{Z} \mu$ under $\pi . B$ is conjugate to a subgroup of $\{1, \theta\} \Delta \mathrm{U}(1)$ or that of $\{1, \theta\} D[4] \otimes \mathrm{U}(n / 2)$ (see $6.2 \mathrm{a}$ and 5.3 ), where $\theta$ is a primitive $2 \mu$ th root of 1. The group $\{1, \theta\} \Delta \mathrm{U}(1)$ is contained in $M$ and gives the value $2^{n}$ for $\#_{2}(M)$ by the above characterization of $M$. We assume that $\mu$ is even. If $n=2$, the group $\{1, \theta\} D[4] \otimes U(n / 2)$ meets $M$ at the subset $\{1, \theta\} \mathrm{I} \otimes \Delta(1)$, where $\mathrm{I}$ is the involutive members of $D[4]$. Thus we have the result for $n=2$. If $n=4$, the group $\{1, \theta\} D[4] \otimes D[4] \otimes \mathrm{U}(1)$ meets $M$ at $\{1, \theta\} \mathrm{I} \otimes \Delta(1)$, where $\mathrm{I}$ is the involutive members of $D[4] \otimes D[4]$. Thus we have the result for $n=4$ by 6.3 .

Proposition 7.2. $\#_{2}(\mathrm{AI}(n) / \mathrm{Z} \mu)$ is 3 if $(n, \mu)=(2,2)$; it is 20 if $(n, \mu)=$ $(4,2)$; it is 10 if $(n, \mu)=(4,4)$; and it is $2^{n-1}$ otherwise.

PROOF. The rank of $\mathrm{AI}(n)$ is $n-1$, and $M^{\prime \prime}:=\mathrm{AI}(n) / Z \mu$ is a subspace of $\mathrm{SU}(n) / \mathbf{Z} \mu$. Thus $2^{n-1} \leq \#_{2} M^{\prime \prime} \leq \#_{2} \mathrm{SU}(n) / \mathbf{Z} \mu$ by 2.7 . If $(n, \mu)=(2,2)$, then $M$ is $\left(S^{2}\right)^{*}$. If $(n, \mu)=(4,2)$, then $M$ is $G_{3}\left(\mathbf{R}^{6}\right)$. If $(n, \mu)=(4,4)$, then $M$ is $G_{3}\left(\mathbf{R}^{6}\right)^{*}$. Hence we have the conclusions in these cases by 6.1 and 6.2 . The other cases are immediate from 5.10 and the above inequalities.

Proposition 7.3. \# $\#_{2}(\mathrm{UII}(n) / \mathbf{Z} \mu)$ is 12 if $n=2$ and $\mu$ is even; it is 56 if $n=4$ and $\mu$ is even; it is 272 if $n=8$ and $\mu$ is even; and it is $2^{n}$ otherwise.

Proof. $M:=\mathrm{UII}(n)=\mathrm{U}(2 n) / \mathrm{Sp}(n)$ has rank $n ; 2^{n} \leq \#_{2}\left(M^{\prime \prime}\right)$, where $M^{\prime \prime}=$ $M / \mathbf{Z} \mu$. The polars of $M^{\prime \prime}$ are $G_{d}\left(\mathbf{H}^{n}\right), 0<d \leq n$, where $G_{d}\left(\mathbf{H}^{n}\right)$ should be replaced with $2 \times G_{d}\left(\mathbf{H}^{n}\right)^{*}$ in case $2 d=n$ and $\mu$ is even. Thus we have $\#_{2}\left(M^{\prime \prime}\right)=2^{n}$ by $6.1,6.4$ and 1.9 , unless $\mu$ is even and $n$ is $2,4,6$ or $8 . M$ is the subspace $F(\iota \circ \kappa \circ \operatorname{ad}(J), \mathrm{U}(2 n))$ of $\mathrm{U}(2 n)$. We assume that $\mu$ is even. The polars of $M^{\prime \prime}$, one of the two components of $F(\iota \circ \kappa \circ \operatorname{ad}(J), \mathrm{U}(2 n) / \mathrm{Z} \mu)$, are the polars $\neq \mathrm{CI}(n)^{*}$ of $\{1, \pi(\theta)\} \operatorname{Sp}(n)^{*}=F(\kappa \circ \operatorname{ad}(J), \mathrm{U}(2 n) / \mathrm{Z} \mu)$, where $\pi$ is the projection: $\mathrm{U}(2 n) \rightarrow$ $\mathrm{U}(2 n) / \mathbf{Z} \mu$. Let $B$ be the inverse image of a maximal antipodal set $A_{2}$ in $M^{\prime \prime}$ under $\pi$. Then the cardinality of $A_{2}$ equals that of $B \cap \operatorname{Sp}(n)$, since $\theta B=B$ (see 5.3) and hence $A_{2}=\{1, \pi(\theta)\}(B \cap \operatorname{Sp}(n))$ (compare 5.4 and 5.8). Also every member of $B \cap \operatorname{Sp}(n)$ is involutive. We can use the groups in the proof of 6.4 for $G_{m}\left(\mathbf{C}^{2 m}\right)^{*}$. If $n=2$, one can use 6.3 and the group $Q[8] \otimes D[4]$ which contains $B$ in order to 
get the 2-number $12=2(1+5)$. If $n=4$, the group $Q[8] \otimes Q[8] \otimes Q[8]$ to get $56=2(1+27)$. If $n=8$, the group $Q[8] \otimes Q[8] \otimes D[4] \otimes D[4]$ gives $2(1+135)=272$. If $n=6$, no group gives a value greater than the regular value.

PROPOSITION 7.4. \# $(\mathrm{AII}(n) / Z \mu)$ is 6 if $(n, \mu)$ is $(2,2)$; it is 28 if $(n, \mu)=$ $(4,4)$; it is 56 if $(n, \mu)=(4,2)$; it is 136 if $(n, \mu)=(8,8)$; it is 272 if $(n, \mu)=(8,4)$ or $(8,2)$; and it is $2^{\text {n-1 }}$ otherwise.

ProOF. Let $M^{\prime \prime}=\operatorname{AII}(n) / \mathbf{Z} \mu$. If $(n, \mu)=(2,2)$, then $M^{\prime \prime}$ is $\left(S^{5}\right)^{*}$. One can proceed as in the proof of 7.3. The polars of $M^{\prime \prime}$ are $G_{d}\left(\mathbf{H}^{n}\right), 0<d=$ even $\leq n$, if $\mu$ is odd; they are $G_{d}\left(\mathbf{H}^{n}\right), 0<d=$ even $\leq n, G_{d}\left(\mathbf{H}^{n}\right)$ replaced with $2 \times G_{d}\left(\mathbf{H}^{n}\right)^{*}$, if $\mu$ and $\nu:=n / \mu$ are even; and they are $G_{d}\left(\mathbf{H}^{n}\right)^{*}, 0<2 d \leq n$ if $\mu$ is even and $\nu$ is odd. Since the rank of $M^{\prime \prime}$ is $n-1$, we thus have the stated 2-numbers by 6.4 and 1.9 except when $\mu$ is even and $n=2,4,6$ or 8 . If $\nu$ is odd, one has $2 \#_{2}\left(M^{\prime \prime}\right)=\#_{2}(\mathrm{UII}(n) / \mathbf{Z} \mu)$, since $M^{\prime \prime}$ meets $\operatorname{Sp}(n)^{*}$ only (and not $\pi(\theta) \operatorname{Sp}(n)^{*}$ which is not contained in $\mathrm{SU}(2 n)$ ). So we assume that both $\mu$ and $\nu$ are even. Then $F(\iota \circ \kappa \circ \operatorname{ad}(J), \mathrm{SU}(2 n))$ is the disjoint union of $M$ and $1_{2} \otimes I_{1}$ times $M$. If $n=4$, the group $Q[8] \otimes Q[8] \otimes Q[8]$ in $\mathrm{Sp}(4)$ meets $F(\iota \circ \kappa \circ \operatorname{ad}(J), \mathrm{SU}(2 n))$ in the first component $M$ only and hence the 2 -number is 56 as in 7.3. If $n=6$, no group gives a value greater than the regular value. If $n=8$, the group $Q[8] \otimes Q[8] \otimes D[4] \otimes D[4]$ in $\mathrm{Sp}(8)$ meets $M$ only as in the case of $n=4$ and hence gives $2(1+135)=272$.

Now we turn to $\operatorname{Spin}(n)$ and related spaces. Recall that $\operatorname{Spin}(n)$, the spinor group, is a subset of the Clifford algebra $\mathrm{CI}(n)$, which is generated over $\mathbf{R}$ by the vectors $\mathbf{e}_{i}$ in the fixed orthonormal basis of $\mathbf{R}^{n}$ and subject to the conditions $\mathbf{e}_{i} \mathbf{e}_{j}=-\mathbf{e}_{j} \mathbf{e}_{i}$ and $\mathbf{e}_{i} \mathbf{e}_{i}=-1, i \neq j$; see [ABS] or [C]. Under the projection $\pi: \operatorname{Spin}(n) \rightarrow \operatorname{SO}(n)$, the member $\cos \theta+(\sin \theta) \mathbf{e}_{1} \mathbf{e}_{2}$ of $\operatorname{Spin}(n)$, for instance, projects to the rotation of the $\left(\mathbf{e}_{1}, \mathbf{e}_{2}\right)$-plane by the angle $2 \theta$, carrying $\mathbf{e}_{1}$ into $(\cos 2 \theta) \mathbf{e}_{1}+(\sin 2 \theta) \mathbf{e}_{2}$, for every real number $\theta . \pi$ is a double covering homomorphism with the kernel $\{1,-1\}$. Every maximal antipodal group $A_{2}$ in $\operatorname{Spin}(n)$ projects into a diagonalizable subgroup of $\mathrm{SO}(n)$. Hence we may assume that $A_{2}$ is a subgroup of $E(n):=\left\{ \pm \mathbf{e}_{I}: I \subset((n))\right\}$, where $\mathbf{e}_{I}=\mathbf{e}_{i} \mathbf{e}_{j} \cdots \mathbf{e}_{k}$ (=1 if $I$ is empty) for any subset $I=\{i, j, \ldots, k\}, i<j<\cdots<k$, of $((n)):=\{1,2, \ldots, n\}$. The next lemma is basic and quite easy to see; it reduces the problem of finding $\#_{2}(\operatorname{Spin}(n))$ to a combinatorial one.

LEMMA 7.5. (i) One has $\mathbf{e}_{I} \mathbf{e}_{J}=\mathbf{e}_{J} \mathbf{e}_{I}$ if and only if the cardinalities satisfy $(\# I)(\# J)-\#(I \cap J) \equiv 0(\bmod 2)$; (ii) one has $\left(\mathbf{e}_{I}\right)^{2}=1$ if and only if \#I $\equiv 0$ or $3(\bmod 4)$; and (iii) $\mathbf{e}_{I}$ is a member of $\operatorname{Spin}(n)$ if and only if \#I is even.

Using this one can determine the 2-rank of $\operatorname{Spin}(n)$. The result is known, which we state it as a proposition without proof; find the number $n-h$ from the table in [Q2].

PROPOSITION 7.6. $r_{2}(\operatorname{Spin}(n))$ is $r+1$ for $n \equiv-1,0$ or $1(\bmod 8)$ and is $=r$ otherwise, where $r$ is the rank of $\operatorname{Spin}(n), r=[n / 2]$.

COROllaRY 7.7 (PERIODICITY). $r_{2}(\operatorname{Spin}(n+8))=r_{2}(\operatorname{Spin}(n))+4$ for $n \geq 0$.

PROPOSITION 7.8. $r_{2}(\operatorname{Pin}(n))=r_{2}(\operatorname{Spin}(n+1))$ for $n \geq 0$.

PrOOF. Pin $(n)$ projects onto $\mathrm{O}(n)$ by definition, [ABS]. $\operatorname{Pin}(n)$ is a group in $\mathrm{CI}(n)$ and contains $\operatorname{Spin}(n) ; \operatorname{Pin}(0)=\operatorname{Spin}(0) \cong \operatorname{Spin}(1) \cong \mathbf{Z}_{2}$ and $\operatorname{Pin}(1)=$ $\left\{1, \mathbf{e}_{1}\right\} \cong \mathbf{Z}_{4}$. Let $A_{2}$ be a great 2 -subgroup of $\operatorname{Pin}(n)$. We may assume that $A_{2}$ is 
a subgroup of $E(n)$, the inverse image of the group $\Delta(n)$ of the diagonal matrices in $\mathrm{O}(n)$ under the projection. If a member $\mathbf{e}_{I}$ of $A_{2}$ belongs to $\operatorname{Spin}(n)$, then it is a member of $\operatorname{Spin}(n+1)$ in the natural fashion. If not, $\mathbf{e}_{I} \mathbf{e}_{n+1}$ is a member of

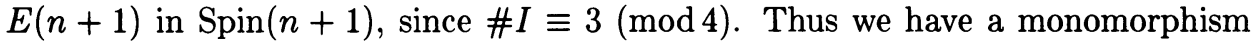
of $A_{2}$ into $\operatorname{Spin}(n+1) ; r_{2}(\operatorname{Pin}(n)) \leq r_{2}(\operatorname{Spin}(n+1))$. One obtains the reversed inequality by reversing the argument.

Proposition 7.9. Let $\mathrm{SO}(4 m)^{\#}$ denote $\operatorname{Spin}(4 m) /\left\{1, \mathbf{e}_{((4 m))}\right\}$ the semispinor group. Then $r_{2}\left(\mathrm{SO}(4 m)^{\#}\right)$ is 3 for $m=1$; it is 6 for $m=2$; it is $r+1$ if $m$ is even $>2$; and it is $r$ if $m$ is odd $>1$, where $r$ is the rank $2 m$ of $\mathrm{SO}(4 m)^{\#}$.

ProOF. Given an antipodal subgroup $A_{2}$ of $\mathrm{SO}(4 m)^{\#}$, we write $B$ for the inverse image of $A_{2}$ under the projection: $\operatorname{Spin}(4 m) \rightarrow \mathrm{SO}(4 m)^{\#}$. All the members $x, y, \ldots$ of the projection $\pi(B) \subset \mathrm{SO}(4 m)$ satisfy $x^{2}= \pm 1$ and $y x= \pm x y$. In particular, $\pi(B)$ is a subgroup of $\mathrm{SO}(4 \mathrm{~m})$ mentioned in $6.2 \mathrm{a}$; it is conjugate to a subgroup of $\Delta, \mathrm{U}(2 m), D[4] \otimes \mathrm{O}(2 m)$ or $Q[8] \otimes \mathrm{Sp}(m)$. If $m$ is odd, consider the group $B_{m}$ generated in $\operatorname{Spin}(4 m)$ by $J^{\prime}:=2^{-m} \prod\left(1+\mathbf{e}_{i} \mathbf{e}_{i+2 m}\right)$, product over $1 \leq i \leq 2 m$, and $\left\{\mathbf{e}_{P I} ; I \subset((2 m))\right\}$, where $P I$ denotes the union $I \cup\{i+2 m \mid i \in I\}$. $\pi\left(B_{m}\right)$, a subgroup of $\mathrm{U}(2 m)$, satisfies those conditions, and the projection of $B_{m}$ into $\mathrm{SO}(4 m)^{\#}$ is an antipodal group of 2-rank $=r$. If $m$ is even, the subgroup $B$ generated by $B_{m}$ and $\mathbf{e}_{((2 m))}$ projects to $A_{2}$ of 2 -rank $r+1$. If $m$ is 1 or 2 , then the group $\pi(B)=\{1, i\} D[4]$ or $\{1, i\} D[4] \otimes D[4]$ (which gives the 2-rank of $\mathrm{U}(2 m) / \mathbf{Z}_{2}$ for $m=1$ or 2 ; cf. 5.2 ) gives the stated 2-ranks.

The rest of the proof is more or less similar to previous ones and omitted.

8. Exceptional spaces. The 2-numbers of $G_{2}$ and $\mathrm{GI}:=G_{2} / \mathrm{SO}(4)$ have been determined in 3.13 .

LEMMA 8.1. One has $r_{2}(G) \leq r_{2}(\operatorname{Sp}(1) \cdot G) \leq 2+r_{2}(G)$ for a compact Lie group $G$ with a pole. (The proof establishes a more precise fact, which will be summarized as Lemma 8.1a.)

ProOF. Let $B$ be the inverse image of a great antipodal group in $\operatorname{Sp}(1) \cdot G$ under the projection: $\operatorname{Sp}(1) \times G \rightarrow \operatorname{Sp}(1) \cdot G$. There are three cases to distinguish. If $B$ is antipodal, then $B$ is great and hence

$$
r_{2}(\operatorname{Sp}(1) \cdot G)=r_{2}(B)-1=r_{2}(\operatorname{Sp}(1) \times G)-1=r_{2}(\operatorname{Sp}(1))+r_{2}(G)-1=r_{2}(G)
$$

If $B$ is not antipodal but abelian, then

$$
r_{2}(\operatorname{Sp}(1) \cdot G)=r_{2}(\mathrm{U}(1) \cdot G)=r_{2}\left(\mathbf{Z}_{4} \cdot G\right)=r_{2}\left(\mathbf{Z}_{4} \cdot F(\operatorname{ad}(i), G)\right),
$$

where $\mathbf{Z}_{4}$ is the cyclic group $\{ \pm 1, \pm i\}$ generated by $i$ in the circle group $\mathrm{U}(1)=$ $T$. Thus 3.14 applies to $\mathrm{U}(1) \cdot G$, or alternatively one sees $r_{2}(\operatorname{Sp}(1) \cdot G)=1+$ $r_{2}(F(\operatorname{ad}(i), G))$, since $B$ meets $F(\operatorname{ad}(i), G)$ at its great antipodal subgroup. Hence $r_{2}(\operatorname{Sp}(1) \cdot G)=1+r_{2}(G)$ if $r_{2}(F(\operatorname{ad}(i), G))=r_{2}(G) ;$ and it equals $r_{2}(G)$ otherwise, since $r_{2}(\operatorname{Sp}(1) \cdot G) \geq r_{2}(G)$ anyway by 3.9 . Finally, if $B$ is not abelian, $B$ contains members $(i, x)$ and $(j, y)$ such that $\{i, j\}$ generates a subgroup $\cong Q[8]$ in $\operatorname{Sp}(1)$ (never $\cong D[4])$. Hence $\{x, y\}$ generates a subgroup $\cong Q[8]$ in $G$, which also we denote by $Q[8]$. Thus $B$ is isomorphic with a subgroup of $Q[8] \cdot F(\operatorname{ad}(Q[8]), G)$; one has $r_{2}(\operatorname{Sp}(1) \cdot G)=r_{2}(Q[8] \cdot F(\operatorname{ad}(Q[8]), G))$. Since $F(\operatorname{ad}(Q[8]), G)$ identified with 
$\{1\} \times F(\operatorname{ad}(Q[8]), G)$ in $\operatorname{Sp}(1) \times G$ projects into $\operatorname{Sp}(1) \cdot G$ injectively, $B$ meets it at its great antipodal subgroup. Therefore $r_{2}(\operatorname{Sp}(1) \cdot G)=2+r_{2}(F(\operatorname{ad}(Q[8]), G))$. Thus $r_{2}(\operatorname{Sp}(1) \cdot G)=2+r_{2}(G)$ if $r_{2}(F(\operatorname{ad}(Q[8]), G))=r_{2}(G) ;$ and $r_{2}(G) \leq r_{2}(\operatorname{Sp}(1) \cdot G) \leq$ $1+r_{2}(G)$ otherwise.

LEMMA 8.1a. Let $G$ be a compact Lie group with a pole $p$. Consider the dot product $\operatorname{Sp}(1) \cdot G$ defined with $p$. Then the 2-rank $r_{2}(\operatorname{Sp}(1) \cdot G)$ has the values as follows. (i) $r_{2}(\operatorname{Sp}(1) \cdot G)=2+r_{2}(G)$ if $G$ has a subgroup (which is isomorphic with) $Q[8]$ such that the center of $Q[8]$ is $\{1, p\}$ and $r_{2}(F(\operatorname{ad}(Q[8]), G))=r_{2}(G)$; (ii) $r_{2}(\operatorname{Sp}(1) \cdot G)=1+r_{2}(G)$ if $G$ has a member $i$ such that $i^{2}=p$ and $r_{2}(F(\operatorname{ad}(i), G))=$ $r_{2}(G)$; and (iii) $r_{2}(\operatorname{Sp}(1) \cdot G)=r_{2}(G)$ if $G$ does not have such a member as $i$.

ProOF. (i) and (ii) are obvious from the above proof of 8.1. Suppose $G$ does not have such a member as $i$. Then $G$ does not have such a subgroup as $Q[8]$, for otherwise one would have $r_{2}(F(\operatorname{ad}(i), G)) \geq r_{2}(F(\operatorname{ad}(Q[8]), G))=r_{2}(G)$, where $\{1, i\}$ is the center of $Q[8]$. The subgroup $B$ in the proof of 8.1 is thus either antipodal in $\operatorname{Sp}(1) \times G$ or abelian (without being antipodal). In the first case, one has $r_{2}(\operatorname{Sp}(1) \cdot G)=r_{2}(G)$, and in the second case, one has $r_{2}(\operatorname{Sp}(1) \cdot G)=$ $1+r_{2}(F(\operatorname{ad}(i), G))$ with $r_{2}(F(\operatorname{ad}(i), G))<r_{2}(G)$ and one still concludes that $r_{2}(\operatorname{Sp}(1) \cdot G)=r_{2}(G)$, since $r_{2}(\operatorname{Sp}(1) \cdot G) \geq r_{2}(G)$ always by 3.9 .

Proposition 8.2. One has $r_{2}\left(G_{2}\right)=3, r_{2}\left(F_{4}\right)=5, r_{2}\left(E_{6}\right)=6, r_{2}\left(E_{7}\right)=7$, $r_{2}\left(E_{8}\right)=9$ for the 1-connected exceptional groups.

PrOOF. We will use 8.1 and 2.13. We have $r_{2}\left(G_{2}\right)=r_{2}(\mathrm{SO}(4))=3$, since $\mathrm{SO}(4)$ is the only group that is orthogonal to the polar. In $F_{4}, \operatorname{Sp}(1) \cdot \operatorname{Sp}(3)$ and $\operatorname{Spin}(9)$ are orthogonal to the polars. Thus we have $r_{2}\left(F_{4}\right)=5$ by 8.1 and 7.6 . In $E_{6}, T \cdot \operatorname{Spin}(10)$ and $\mathrm{Sp}(1) \cdot \mathrm{SU}(6)$ are orthogonal to the polars. We see $r_{2}(T \cdot \operatorname{Spin}(10)) \leq 6$ by 3.11 . We have $r_{2}(\mathrm{Sp}(1) \cdot \mathrm{SU}(6)) \leq 6$ by 8.1 , since $r_{2}(F(\operatorname{ad}(Q[8]), \mathrm{SU}(6)))=r_{2}(\mathrm{U}(3))=$ $3<5=r_{2}(\mathrm{SU}(6))$. Thus we have $r_{2}\left(E_{6}\right)=6$. In $E_{7}, \operatorname{Sp}(1) \cdot \operatorname{Spin}(12)$ is the only orthogonal to the polar. This dot product is defined with the kernel of the projection of Spin(12) onto SO(12)\#. In particular, a subgroup $Q[8]$ in the centrosome plus the center in $\operatorname{Spin}(12)$ for this dot product projects onto an isomorphic $Q[8]$ in $\mathrm{SO}(12)$. The centralizer $F(\operatorname{ad}(Q[8]), \operatorname{Spin}(12))$ is $2 \times \operatorname{Sp}(3)$, the union of 2 copies of $\operatorname{Sp}(3)$, and hence its 2-rank is less than $r_{2}(\operatorname{Spin}(12))=6$. Thus we have $r_{2}(\operatorname{Sp}(1) \cdot \operatorname{Spin}(12)) \leq 7$. Hence $r_{2}\left(E_{7}\right)=7$ by 8.1. In $E_{8}, \mathrm{Sp}(1) \cdot E_{7}$ and $\mathrm{SO}(16)^{\#}$ are the orthogonal to the polars. Therefore we obtain $r_{2}\left(E_{8}\right)=9$ by 8.1 and 7.9 .

PROPOSITION 8.3. $r_{2}\left(F_{4}\right)=5, \#_{2}(\mathrm{FI})=28=2^{r}+\chi$ and $\#_{2}(\mathrm{FII})=3=\chi$, where $r$ and $\chi$ are the rank and the Euler number of the space in question.

ProOF. We have $\#_{2}(\mathrm{FI}) \geq 28=2^{r}+\chi$ by 4.2 . The polars of FI are $G_{1}\left(\mathbf{H}^{4}\right)$ and $S^{2} \cdot \mathrm{CI}(3)$. We know \# $\#_{2}\left(G_{1}\left(\mathbf{H}^{4}\right)\right)=4$ by $6.1 . S^{2} \cdot \mathrm{CI}(3)$ is the centrosome of $\mathrm{Sp}(1) \cdot \mathrm{Sp}(3)$. Thus the great antipodal set in $S^{2} \cdot \mathrm{CI}(3)$ is contained in $Q[8] \otimes \Delta(3)$ as in the proof of 5.14 . We obtain $\#_{2}\left(S^{2} \cdot \mathrm{CI}(3)\right)=23$ easily and conclude $\#_{2}(\mathrm{FI})=28$. See 2.11 for $\#_{2}$ (FII) and 8.2 for the rest.

Proposition 8.4. $r_{2}\left(E_{6}\right)=6=r_{2}\left(E_{6}^{*}\right), \#_{2}(\mathrm{EI})=64=2^{r}=\#_{2}\left(\mathrm{EI}^{*}\right)$, $\#_{2}(\mathrm{EII})=36=\chi, \#_{2}(\mathrm{EIII})=27$, and $\#_{2}(\mathrm{EIV})=4=2^{r}=\#_{2}\left(\mathrm{EIV}^{*}\right)$.

PROOF. EIII is hermitian and 4.3 applies. EI has rank 6 and is contained in $E_{6}$, and hence its 2-number is found from 8.2. FII is the only polar of EIV; 
thus $\#_{2}(E I V)=4$ by 2.10 and 8.3 . The fundamental groups of $E_{6}^{*}$ and $\mathrm{EI}^{*}$ have order 3 , and hence their 2-numbers are equal to those of their universal coverings by 3.1. Finally we come to EII, which is a polar of $E_{6}$ together with EIII; we have $\#_{2}$ (EII) $\geq \#_{2}\left(E_{6}\right)-1-27=36$ by 4.2 . The polars of EII are $G_{2}\left(C^{6}\right)$ and $S^{2} \cdot G_{3}\left(C^{6}\right)$. Since $S^{2} \cdot G_{3}\left(C^{6}\right)$ is a centrosome of $\mathrm{Sp}(1) \cdot \mathrm{SU}(6)$, its antipodal set is contained in $\{1, i\} \cdot \Delta(6)$ or $Q[8] \cdot \mathrm{U}(3)$. Both cases give $\#_{2}\left(S^{2} \cdot G_{3}\left(C^{6}\right)\right)=$ $\#_{2}\left(G_{3}\left(C^{6}\right)\right)=20$ and hence $\#_{2}(\mathrm{EII})=36$. The fundamental groups of the spaces with ${ }^{*}$ in the proposition have order 3 and 3.1 applies.

REMARK 8.4a. The orthogonal space of $\mathrm{EI}$ in $E_{6}$ is $\mathrm{Sp}(4)^{*}$. Hence we rediscover its 2-rank $=6$ (see 5.14).

Proposition 8.5. $r_{2}\left(E_{7}\right)=7, \#_{2}(\mathrm{EV})=128=2^{r}, \#_{2}(\mathrm{EVI})=63=\chi$, and $\#_{2}(\mathrm{EVII})=56=\chi$.

ProOF. EV is a subspace of $E_{7}$ and of the same rank; thus $\#_{2}(\mathrm{EV})=128$ (also by 3.6 and 6.4). EVII is hermitian; 4.3 applies. The polars of $E_{7}$ are a polar and 2 copies of EVI. The double covering: $E_{7} \rightarrow E_{7}^{*}$ carries both copies onto a single EVI. Thus $\#_{2}(E V I)=\left(\#_{2}\left(E_{7}\right)-2\right) / 2=63$ by 3.6 .

Appendix. For the reader's convenience, we will record the fixed point sets $F(t, M)$ of the involutive automorphisms $t$ of the compact connected symmetric spaces $M$, most of which are assumed to be irreducible; the results are quoted from $[\mathbf{C N}],[\mathbf{N}]$ and subsequent papers.

We begin with the special case in which $t$ is the symmetry at a point; so the connected components of $F(t, M)$ are its polars. T'hose spaces that have the same roots (with different multiplicity) have the same and obvious pattern of the polars and $F(t, M)$ more generally, and hence we omit some of the spaces which are locally isomorphic.

Spaces The polars

torus $T^{r} \quad 2^{r}-1$ poles.

$\mathrm{U}(n) / \mathbf{Z} \mu \quad G_{r}\left(\mathbf{C}^{n}\right), 0<r \leq n$; if $\mu$ is even and $2 r=n$, then $G_{r}\left(\mathbf{C}^{n}\right)$ is replaced by 2 copies $2 \times G_{r}\left(\mathbf{C}^{2 r}\right)^{*}$ of $G_{r}\left(\mathbf{C}^{2 r}\right)^{*}$.

$\mathrm{SU}(n) / \mathbf{Z} \mu \quad G_{r}\left(\mathbf{C}^{n}\right), 0<r=$ even $\leq n$, if $\mu$ is odd.

$G_{r}\left(\mathbf{C}^{n}\right)^{*}, 0<2 r \leq n$, if $\mu$ is even and $\nu:=n / \mu$ is odd.

$G_{r}\left(\mathbf{C}^{n}\right)^{*}, 0<r=$ even $\leq n$, if $\mu$ and $\nu$ are even ; $G_{r}\left(\mathbf{C}^{n}\right)^{*}$

$\mathrm{SO}(n) \quad G_{r}\left(\mathbf{R}^{n}\right), 0<r=$ even $\leq n$.

$\operatorname{Spin}(n) \quad G_{2 r}^{o}\left(\mathbf{R}^{n}\right), 0 \leq r=$ even $\leq n / 2$, with the understanding that

this is a single point for $r=0$ and 2 points for $r=n / 2$.

$\mathrm{SO}(n)^{\#}, \quad \operatorname{OIII}(n / 2)^{*}$ and $G_{2 r}^{o}\left(\mathbf{R}^{n}\right), 0 \leq r=$ even $\leq n / 4$, with the under-

$n \equiv 0 \bmod 4$ standing that this is a single point for $r=0$ and $G_{2 r}^{o}\left(\mathbf{R}^{n}\right)$

is replaced with $G_{2 r}\left(R^{n}\right)^{\#}$ for $r=n / 4$.

$\mathrm{SO}(n)^{*}, \quad G_{r}\left(\mathbf{R}^{n}\right)^{*}, 0<r=$ even $\leq n$, and $\operatorname{DIII}(n / 2)$. Replace $\operatorname{DIII}(n / 2)$

$n$ even. with $\operatorname{OIII}(n / 2)^{*}$ if $n / 2$ is even.

$\operatorname{Sp}(n) \quad G_{r}\left(\mathbf{H}^{n}\right), 0<r \leq n$.

$\operatorname{Sp}(n)^{*} \quad G_{r}\left(\mathbf{H}^{n}\right)^{*}, 0<2 r \leq n$, and $\mathrm{CI}(n)^{*}$. 


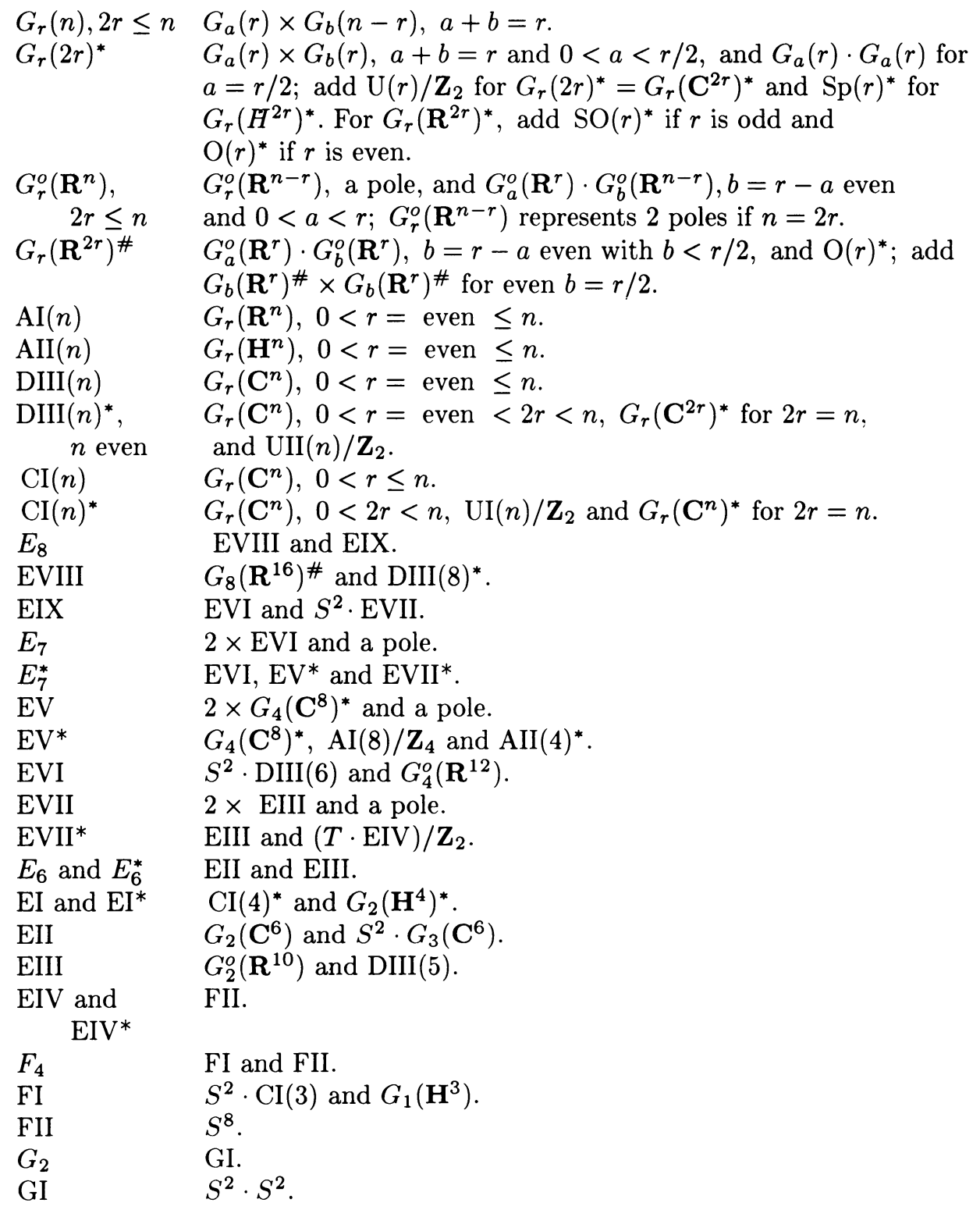

Next we describe $F(t, M)$ for each conjugate class of the involutions $t$ of $M$, which fix a point $o$ of $M$. We omit the case of the groups; since the results for them are essentially the classification of the symmetric spaces. We select only one space from its local isomorphism class to keep the table reasonably short.

We begin with the spaces $M=G / K$ with $G$ locally isomorphic with $\operatorname{SU}(n)$. Now $t$ on $M$ is induced from one of the conjugate classes of the involutions of $\operatorname{SU}(n)$, ${ }^{s} \mathrm{I}:=\kappa,{ }^{s} \mathrm{II}:=\kappa \circ \operatorname{ad}(J)$, and $\mathrm{I}_{d}$; see the Introduction for these notations, where $M$ is $\mathrm{AI}(n)$ identified with $F\left(s_{1} \circ \kappa, \mathrm{SU}(n)\right), \operatorname{AII}(n)$ with $F\left(s_{1} \circ \kappa \circ \operatorname{ad}(J)_{(1)}, \mathrm{SU}(2 n)\right)$, or $G_{r}\left(\mathbf{C}^{n}\right)$ with $F\left(s_{1} \circ \operatorname{ad}\left(\mathrm{I}_{r}\right), \mathrm{SU}(n)\right)$. We distinguish an involution $b$ from $\operatorname{ad}(b)$; $b$ on $M=G / K$ carries $c K$ into $b c K$, while $\operatorname{ad}(b)$ carries $c K$ into $(\operatorname{ad}(b) c) K$, which 
coincides with $b$ in case $b$ is a member of $K$ and which can be defined even if $b$ is not a member of $G$ but a member of a larger group. $P_{d}$ means $\mathrm{I}_{d} \oplus \mathrm{I}_{d}$, and similarly $P_{J}=J \oplus J$.

\begin{tabular}{|c|c|c|c|}
\hline & $\mathrm{AI}(n)$ & $\operatorname{AII}(n)$ & $G_{r}\left(\mathbf{C}^{n}\right)$ \\
\hline${ }^{8} \mathrm{I}$ & (polars) & $\operatorname{DIII}(n)$ & $G_{r}\left(\mathbf{R}^{n}\right)$ \\
\hline$s_{o} \circ{ }^{s} \mathrm{I}$ & & $\mathrm{SU}(n)$ & $G_{r}\left(\mathbf{R}^{n}\right)$ \\
\hline${ }^{s} \mathrm{II}$ & $2 \times \mathrm{SU}(n / 2)$ & (polars) & $G_{r / 2}\left(\mathbf{H}^{n / 2}\right)$ \\
\hline$s_{o} \circ{ }^{s} \mathrm{II}$ & $\mathrm{CI}(n / 2)$ & & $G_{r / 2}\left(\mathbf{H}^{n / 2}\right)$ \\
\hline $\mathrm{I}_{d}$ & $T \cdot(\operatorname{AI}(d) \times \operatorname{AI}(n-d))$ & $T \cdot(\operatorname{AII}(d) \times \operatorname{AII}(n-d))$ & $\begin{array}{c}G_{a}\left(\mathbf{C}^{d}\right) \times G_{b}\left(\mathbf{C}^{n-d}\right) \\
\quad \text { II for } a+b=r .\end{array}$ \\
\hline$s_{o} \circ \operatorname{ad}\left(I_{1}\right)$ & $\amalg_{k \text { odd }}^{G} G_{k}\left(\mathbf{R}^{n}\right)$ & $\coprod_{k \text { odd }}^{G_{k}\left(\mathbf{H}^{n}\right)}$ & \\
\hline
\end{tabular}

\begin{tabular}{lcc} 
& $\mathrm{DIII}(n)$ & $G_{r}\left(\mathbf{R}^{n}\right)$ \\
\hline $\mathrm{I}_{d}$ & & $G_{a}\left(\mathbf{R}^{d}\right) \times G_{b}\left(\mathbf{R}^{n-d}\right)$ \\
& & $\amalg$ for $a+b=r$. \\
$P_{d}$ & $2 \times(\operatorname{DII}(d) \times \operatorname{DII}(n-d))$ & \\
$J_{r / 2} \oplus J_{n / 2-r / 2}$ & $0 \leq 2 d \leq n$ & $G_{r / 2}\left(\mathbf{C}^{n / 2}\right)$ \\
$\operatorname{ad}(J)$ & $($ polars $)$ & $\mathrm{UI}(r)$ if $n=2 r$ \\
$s_{o} \circ \operatorname{ad}(J)$ & & $\mathrm{O}(r)$ if $n=2 r$ \\
$\operatorname{ad}\left(\mathrm{I}_{n}\right)$ & $\mathrm{SO}(n)$ & \\
$J$ & $\bigsqcup G_{k}\left(\mathbf{C}^{n}\right), k$ even \\
$J P_{1}$ & $\bigsqcup G_{k}\left(\mathbf{C}^{n}\right), k$ odd \\
$\operatorname{ad}\left(P_{J} \mathrm{I}_{n}\right)$ & $\mathrm{UII}(n / 2)$
\end{tabular}

\begin{tabular}{lcc} 
& $\mathrm{CI}(n)$ & $G_{r}\left(\mathbf{H}^{n}\right)$ \\
\hline$P_{d}$ & $\mathrm{CI}(d) \times \mathrm{CI}(n-d)$ & $G_{a}\left(H^{d}\right) \times G_{b}\left(\mathbf{H}^{n-d}\right)$ \\
$\operatorname{ad}\left(\mathrm{I}_{n}\right)$ & $\mathrm{UI}(n)$ & $\coprod$ for $a+b=r$. \\
$\operatorname{ad}\left(P_{J} K\right)$ & $\mathrm{Sp}(n / 2)$ & \\
$J$ & $($ polars $)$ & $G_{r}\left(\mathbf{C}^{n}\right)$ \\
$\operatorname{ad}\left(P_{J}\right)$ & & $\mathrm{UII}(n)$ \\
$\boldsymbol{s}_{o} \circ \operatorname{ad}\left(P_{J}\right)$ & & $\mathrm{Sp}(n)$
\end{tabular}


${ }^{s} \mathrm{I},{ }^{s} \mathrm{II}, \ldots$ will denote the involutive members of $E_{6}, \ldots$ which define the spaces EI, EII,..., except that ${ }^{s} \mathrm{I}$ and ${ }^{s} \mathrm{IV}$ are outer involutive automorphisms which define EI and EIV.

\begin{tabular}{|c|c|c|c|c|}
\hline & EI & EII & EIII & EIV \\
\hline${ }^{s} \mathbf{I}$ & $\mathrm{CI}(4)^{*} \coprod G_{2}\left(\mathbf{H}^{4}\right)^{*}$ & $\mathrm{CI}(4)^{*} \coprod G_{1}\left(\mathbf{H}^{4}\right)$ & $G_{2}\left(\mathbf{H}^{4}\right)^{*}$ & $G_{1}\left(\mathbf{H}^{4}\right)$ \\
\hline${ }^{s} \mathrm{II}$ & $S^{2} \cdot \operatorname{AI}(6) \coprod \mathrm{AII}(3)$ & $S^{2} \cdot G_{3}\left(\mathbf{C}^{6}\right) \coprod G_{2}\left(\mathbf{C}^{6}\right)$ & $S^{2} \times G_{1}\left(\mathbf{C}^{6}\right) \bigsqcup G_{2}\left(\mathbf{C}^{6}\right)$ & $\operatorname{AII}(3)$ \\
\hline${ }^{s}$ III & $T \cdot G_{5}\left(\mathbf{R}^{10}\right)$ & $G_{4}^{o}\left(\mathbf{R}^{10}\right) \coprod_{\mathrm{FI}} \mathrm{DIII}(5)$ & $G_{2}^{o}\left(\mathbf{R}^{10}\right) \coprod_{\mathrm{FII}} \operatorname{DIII}(5)$ & $T \cdot S^{9}$ \\
\hline
\end{tabular}

In the next table, " will mean $/ \mathbf{Z}_{2}$.

\begin{tabular}{|c|c|c|c|}
\hline & EV & EVI & EVII \\
\hline $\operatorname{ad}\left({ }^{s} \mathrm{~V}\right)$ & $\mathrm{AI}(8)^{\prime \prime}\left[\mathrm{AII}(4)^{\prime \prime}\right.$ & & $\operatorname{AII}(4)^{\prime \prime}$ \\
\hline${ }^{s} \mathrm{~V}$ & $2 \times G_{4}\left(\mathbf{C}^{8}\right)^{*}$ & $G_{4}\left(\mathbf{C}^{8}\right)^{*} \coprod G_{2}\left(\mathbf{C}^{8}\right)$ & $2 \times G_{2}\left(\mathbf{C}^{8}\right)$ \\
\hline${ }^{s} \mathrm{VI}$ & $S^{2} \cdot G_{6}^{o}\left(\mathbf{R}^{12}\right) \coprod \mathrm{DIII}(6)$ & $S^{2} \cdot \operatorname{DIII}(6) \coprod G_{4}^{o}\left(\mathbf{R}^{12}\right)$ & $S^{2} \times G_{2}^{o}\left(\mathbf{R}^{12}\right) \coprod \operatorname{DIII}(6)$ \\
\hline $\begin{array}{l}{ }^{s} \mathrm{VII} \\
\operatorname{add}\left({ }^{s} \mathrm{VII}\right)\end{array}$ & $\begin{array}{c}2 \times \mathrm{EII} \\
T \cdot \mathrm{EI}\end{array}$ & EII ЦEIII & $\begin{array}{l}2 \times \mathrm{EIII} \\
T \cdot \mathrm{EIV}\end{array}$ \\
\hline
\end{tabular}

\begin{tabular}{lcc} 
& EVIII & EIX \\
\hline${ }^{s}$ VIII & $G_{8}\left(\mathbf{R}^{16}\right) \# \coprod \mathrm{DIII}(8)^{*}$ & $G_{4}^{o}\left(\mathbf{R}^{16}\right) \coprod \mathrm{DIII}(8)^{*}$ \\
${ }^{s} \mathrm{IX}$ & $S^{2} \cdot \mathrm{EV} \coprod \mathrm{EVI}$ & $S^{2} \cdot \mathrm{EVII} \coprod \mathrm{EVI}$
\end{tabular}

${ }^{s} \mathrm{I}$ and ${ }^{s}$ II will denote the involutive members of $F_{4}$ which define the spaces FI and FII.

\begin{tabular}{ccc} 
& FI & FII \\
\hline${ }^{s} \mathrm{I}$ & $S^{2} \cdot \mathrm{CI}(3) \coprod_{{ }^{3}} G_{1}\left(\mathbf{H}^{3}\right)$ & $G_{1}\left(\mathbf{H}^{3}\right)$ \\
${ }^{\mathrm{II}}$ & $G_{4}^{o}\left(\mathbf{R}^{9}\right)$ & $S^{8}$
\end{tabular}

\section{BIBLIOGRAPHY}

[ABS] M. F. Atiyah, R. Bott and A. Shapiro, Clifford modules, Topology 3 Suppl. 1 (1964), 3-38. [AS] M. F. Atiyah and I. M. Singer, The index of elliptic operators. III, Ann. of Math. (2) 87 (1968), 546-604.

[B] A. Borel, Sur l'homologie et la cohomologie des groupes de Lie compacts connexes, Amer. J. Math. 76 (1954), 273-342.

[B2] __ Commutative subgroups and torsion in compact Lie groups, Bull. Amer. Math. Soc. 66 (1960), 285-288.

[BSe] A. Borel and J. P. Serre, Sur certains sousgroupes des groupes de Lie compacts, Comm. Math. Helv. 27 (1953), 128-139.

[CN] B. Y. Chen and T. Nagano, Totally geodesic submanifolds of symmetric spaces. II, Duke Math. J. 45 (1978), 405-425.

[CN1] _ Un invariant géométrique Riemannien, C.R. Acad. Sci. Paris 295 (1982), 389--391.

[C] C. Chevalley, The algebraic theory of spinors, Columbia Univ. Press, New York, 1954.

[N] T. Nagano, The involutions of compact symmetric spaces (to appear).

[H] S. Helgason, Differential geometry, Lie groups and symmetric spaces, Academic Press, New York, 1978. 
[HS] H. Hopf and H. Samelson, Ein Satz uber Wirkungsraume geschlossener Liescher Gruppen, Comm. Math. Helv. 13 (1941), 240-251.

[Q] D. Quillen, The spectrum of an equivariant cohomology ring, Ann. of Math. (2) 94 (1971), 549-609.

[Q2] _ The mod 2 cohomology rings of extra-special 2-groups and the spinor groups, Math. Ann. 194 (1971), 197-212.

Department of Mathematics, Michigan State University, East Lansing, MICHIGAN 48824

Department of MAThematics, Sophia UNIVERSity, TOKYO, 102 JAPAN 